\title{
On degenerate coupled transport processes in porous media with memory phenomena
}

\author{
Michal Beneš* and Igor Pažanin ${ }^{\dagger}$
}

\begin{abstract}
In this paper we prove the existence of weak solutions to degenerate parabolic systems arising from the fully coupled moisture movement, solute transport of dissolved species and heat transfer through porous materials. Physically relevant mixed Dirichlet-Neumann boundary conditions and initial conditions are considered. Existence of a global weak solution of the problem is proved by means of semidiscretization in time, proving necessary uniform estimates and by passing to the limit from discrete approximations. Degeneration occurs in the nonlinear transport coefficients which are not assumed to be bounded below and above by positive constants. Degeneracies in transport coefficients are overcome by proving suitable a-priori $L^{\infty}$-estimates based on De Giorgi and Moser iteration technique.
\end{abstract}

\section{Introduction}

Let $\Omega$ be a bounded domain in $\mathbb{R}^{2}, \Omega \in C^{0,1}$ and let $\Gamma_{D}$ and $\Gamma_{N}$ be open disjoint subsets of $\partial \Omega$ (not necessarily connected) such that $\Gamma_{D} \neq \emptyset$ and the $\partial \Omega \backslash\left(\Gamma_{D} \cup \Gamma_{N}\right)$ is a finite set. Let $T \in(0, \infty)$ be fixed throughout the paper, $I=(0, T)$ and $Q_{T}=\Omega \times I$ denotes the space-time cylinder, $\Gamma_{D T}=\Gamma_{D} \times I$ and $\Gamma_{N T}=\Gamma_{N} \times I$. We shall study the following problem in $Q_{T}$ :

$$
\begin{aligned}
\partial_{t}[\phi(x, r) S(p)]= & \nabla \cdot[a(x, p, \vartheta, r) \nabla p]+\alpha_{1} f(x, p, c, \vartheta, r), \\
\partial_{t}[\phi(x, r) S(p) c]= & \nabla \cdot\left[\phi(x, r) S(p) D_{w}(x, p) \nabla c\right] \\
& +\nabla \cdot[c a(x, p, \vartheta, r) \nabla p], \\
\partial_{t}[\phi(x, r) S(p) \vartheta+\varrho(x, r) \vartheta]= & \nabla \cdot[\lambda(x, p, \vartheta, r) \nabla \vartheta] \\
& +\nabla \cdot[\vartheta a(x, p, \vartheta, r) \nabla p]+\alpha_{2} f(x, p, c, \vartheta, r),
\end{aligned}
$$

where

$$
a(x, p, \vartheta, r)=\frac{k(x, r) k_{R}(S(p))}{\mu(\vartheta)} .
$$

*Department of Mathematics, Faculty of Civil Engineering, Czech Technical University in Prague, Thákurova 7, 16629 Prague 6, Czech Republic, E-mail: michal.benes@cvut.cz

${ }^{\dagger}$ Department of Mathematics, Faculty of Science, University of Zagreb, Bijenička 30, 10000 Zagreb, Croatia, E-mail: pazanin@math.hr 
The system introduced above is coupled with an integral condition

$$
r(x, t)=\int_{0}^{t} f(x, p(x, s), c(x, s), \vartheta(x, s), r(x, s)) \mathrm{d} s
$$

and completed by the mixed-type boundary conditions

$$
\begin{aligned}
& p=0, \quad c=0, \quad \vartheta=0 \quad \text { on } \Gamma_{D T}, \\
& \nabla p \cdot \boldsymbol{n}=0, \quad \nabla c \cdot \boldsymbol{n}=0, \quad \nabla \vartheta \cdot \boldsymbol{n}=0 \quad \text { on } \Gamma_{N T}
\end{aligned}
$$

and the initial conditions

$$
p(\cdot, 0)=p_{0}, c(\cdot, 0)=c_{0}, \vartheta(\cdot, 0)=\vartheta_{0} \quad \text { in } \Omega .
$$

The goal of this paper is to study the existence of the so called weak solution to the degenerate fully coupled nonlinear system (1.1)-(1.8). The problem under consideration covers a large range of problems including memory phenomena. Namely, the system (1.1)-(1.8) arises from the coupled moisture movement, transport of dissolved species and heat transfer through the porous system [3, 40]. Equations (1.1) and (1.2) express the mass balance of water and dissolved species, respectively, in porous media and (1.3) represents the balance of heat energy in the porous system. For simplicity, the gravity terms are not included since they do not affect the analysis. For specific civil engineering applications, we refer the reader to e.g. [13, 39]. Our problem has been motivated by doubly nonlinear systems appearing in modelling of chemical reactions, heat transport and mass transfer in early age concrete [31, 32, 33, 46].

In 1.1)-1.8, $p: Q_{T} \rightarrow \mathbb{R}, c: Q_{T} \rightarrow \mathbb{R}, \vartheta: Q_{T} \rightarrow \mathbb{R}$ and $r: Q_{T} \rightarrow \mathbb{R}$ are the unknown functions. In particular, $p$ corresponds to the water pressure, $c$ represents concentration of dissolved species and $\vartheta$ represents the temperature of the complete porous system. Equations (1.1), (1.3) and (1.5) are encountered e.g. in the so called problem of "hydratational heat" when inner moisture sinks and heat sources are of special types. In particular, the intensity of heat sources depends on the amount of heat already developed, $f$ in (1.1) and (1.3) depends on the unknown function $r$ (the so called "hydration degree") given by (1.5). Further, $a: \Omega \times \mathbb{R}^{3} \rightarrow \mathbb{R}$ represents the transport coefficient of the capillary water given by (1.4), where $k$ is the intrinsic permeability, $k_{R}$ denotes the relative permeability of the liquid water and $\mu$ is the dynamic viscosity of the liquid water. $D_{w}: \Omega \times \mathbb{R} \rightarrow \mathbb{R}$ is the capillary water diffusion coefficient, $S: \mathbb{R} \rightarrow \mathbb{R}$ represents degree of saturation of the pores with liquid water, $\phi: \Omega \times \mathbb{R} \rightarrow \mathbb{R}$ is porosity, $\varrho: \Omega \times \mathbb{R} \rightarrow \mathbb{R}$ is the density of solid skeleton in the porous system. Further, $\lambda: \Omega \times \mathbb{R}^{3} \rightarrow \mathbb{R}$ is the thermal conductivity of porous material. $\alpha_{1}$ and $\alpha_{2}$ are material constants. Note that the density of water is assumed to be constant in the model and normalized to one. $\boldsymbol{n}$ is the outward unit normal vector with respect to the boundary of $\Omega$. Finally, $p_{0}: \Omega \rightarrow \mathbb{R}, c_{0}: \Omega \rightarrow \mathbb{R}$ and $\vartheta_{0}: \Omega \rightarrow \mathbb{R}$ are given functions describing initial state of the system.

Typical forms of $S$ and $k_{R}$ can be found e.g. in [16, 17, 18, 40] with applications to water movement in soils or structured rock masses or in [13, 14, 15] concerning transport processes in concrete. It follows that positive functions $S(\cdot)$ and $k_{R}(S(\cdot))$ are typically increasing on $(-\infty, 0)$ and $S^{\prime}(\cdot)$ and $k_{R}(S(\cdot))$ tend to zero as $p \rightarrow-\infty$. 
Hence, (1.1) and (1.2) are degenerate parabolic equations where the degeneracy occurs in both elliptic as well as parabolic parts. The degeneracy in the elliptic part of (1.1) can be transformed only to the parabolic term using the so called Kirchhoff transformation

$$
v:=\kappa(p)=\int_{0}^{p} k_{R}(S(s)) \mathrm{d} s .
$$

The existence of the weak solution $v$ for the resulting transformed problem follows from Alt and Luckhaus [2]. However, due to the degeneracy of the problem $\left(k_{R}\right.$ is not assumed to be bounded below by a positive constant) we are not able to ensure that $p=$ $\kappa^{-1}(v)$ solves the original problem. Therefore we treat directly the doubly degenerate problem (1.1) and omit degeneracies proving $L^{\infty}$-estimates for the solutions of the approximate problems.

A brief bibliographical survey. Nowadays, description of heat, moisture or soluble/nonsoluble contaminant transport in concrete, soil or rock porous matrix is frequently based on time dependent models. Coupled transport processes (diffusion processes, heat conduction, moister flow, contaminant transport or coupled flows through porous media) are typically associated with systems of strongly nonlinear degenerate parabolic partial differential equations of type (written in terms of operators $A, B, F$ )

$$
\partial_{t} B(\boldsymbol{u})-\nabla \cdot A(\boldsymbol{u}, \nabla \boldsymbol{u})=F(\boldsymbol{u}),
$$

where $\boldsymbol{u}$ stands for the unknown vector of state variables. There is no complete theory for such general problems. However, some particular results assuming special structure of operators $A$ and $B$ and growth conditions on $F$ can be found in the literature. Most theoretical results on parabolic systems exclude the case of non-symmetrical parabolic parts [2, 10, 23]. Giaquinta and Modica in [19] proved the local-in-time solvability of quasilinear diagonal parabolic systems with nonlinear boundary conditions (without assuming any growth condition), see also [48]. The existence of weak solutions to more general non-diagonal systems like (1.9) subject to mixed boundary conditions has been proven in [2]. The authors proved an existence result assuming the operator $B$ to be only (weak) monotone and subgradient. This result has been extended in [10], where the authors presented the local existence of the weak solutions for the system with nonlinear Neumann boundary conditions and under more general growth conditions on nonlinearities in $\boldsymbol{u}$. These results, however, are not applicable if $B$ does not take the subgradient structure, which is typical of coupled transport models in porous media. Thus, the analysis needs to exploit the specific structure of such problems. The existence of a local-in-time strong solution for moisture and heat transfer in multi-layer porous structures modelling by the doubly nonlinear parabolic system is provided in [7]. In [47], the author proved the existence of the solution to the purely diffusive hygro-thermal model allowing non-symmetrical operators $B$, but requiring non-realistic symmetry in the elliptic part. In [8, 22], the authors studied the existence, uniqueness and regularity of coupled quasilinear equations modeling evolution of fluid species influenced by thermal, electrical and diffusive forces. In [28, 29, 30], the authors studied a model of specific structure of a heat and mass transfer arising 
from textile industry and proved the global existence for one-dimensional problems in [28, 29] and three-dimensional problems in [30]. In [21], the authors proved the existence of the weak solutions to systems modeling the consolidation of saturated porous media.

In [42, 43], the author proved the local existence of weak solutions to degenerate quasilinear problems, where the coefficient function in front of the time derivative may vanish at a set of zero measure. The main result is proved by means of semidiscretization in time and proving $L^{\infty}$-estimates for approximates in order to omit a growth limitations in nonlinearities and the right hand side.

In [38], the author studied an initial boundary value problem for the nonlinear degenerate parabolic equation of type (1.9) with evolutionary boundary conditions. Existence and uniqueness were established through some discrete schemes combined with parabolic regularization and error estimates for these schemes were presented. In a slightly different form, taking $\phi=1$ and $f=0$ and assuming different degeneration features, problem (1.1)-(1.2) was studied in [35, 36, 37].

From the numerical point of view, scalar degenerate problems similar to (1.1) were treated in [24, 25]. The author proposed a nonstandard approximation scheme based on the relaxation method in order to control the degeneracy in the problem.

A profound investigation of problems with integral conditions connected with equations (1.1) and (1.5) can be found in [9] and [44] in case of nondegenerate linear elliptic and parabolic parts and in [24] assuming strongly nonlinear and degenerate scalar parabolic problems.

Outline of the paper. In the present paper we extend our previous existence result for coupled heat and mass flows in porous media [4] to more general degenerate problem modeling coupled moisture, solute and heat transport in porous media including memory phenomena. This leads to a fully nonlinear degenerate parabolic system coupled with an integral condition and including natural (critical) growths and with degeneracies in transport coefficients. The rest of this paper is organized as follows. In Section 2, we briefly introduce basic notation and suitable function spaces and specify our assumptions on data and coefficient functions in the problem. In Section 3, we formulate the problem in the variational sense and state the main result, the global-in-time existence of the weak solution to the problem (1.1)-(1.8). The main result is proved by an approximation procedure in Section 4 First, we formulate the semi-discrete scheme and prove the existence of the solution to the corresponding recurrence steady problem. The crucial a-priori estimates and uniform boundness of time discrete approximations are proved in Section 4.2 Finally, we conclude that solutions of the semi-discrete scheme converge and that the limit corresponds to the solution of the original problem.

Remark 1.1 The present analysis can be straightforwardly extended to a setting with nonhomogeneous boundary conditions (1.6) (see [4] for details or [2] Paragraph 1.10 on page 324]). Here we work with homogeneous boundary conditions to avoid unnecessary technicalities in the existence result. 


\section{Preliminaries}

\subsection{Notations and some function spaces}

Vectors and vector functions are denoted by boldface letters. Throughout the paper, we will always use positive constants $C, C_{1}, C_{2}, \ldots$, which are not specified and which may differ from line to line. In what follows, we suppose $s, q, s^{\prime} \in[1, \infty], s^{\prime}$ denotes the conjugate exponent to $s>1,1 / s+1 / s^{\prime}=1$. $L^{s}(\Omega)$ represents the usual Lebesgue space equipped with the norm $\|\cdot\|_{L^{s}(\Omega)}$ and $W^{k, s}(\Omega), k \geq 0$ ( $k$ need not to be an integer, see [26]), denotes the usual Sobolev-Slobodecki space with the norm $\|\cdot\|_{W^{k, s}(\Omega)}$. We define $W_{\Gamma_{D}}^{1,2}(\Omega):=\left\{v \in W^{1,2}(\Omega) ;\left.v\right|_{\Gamma_{D}}=0\right\}$. By $E^{*}$ we denote the space of all continuous, linear forms on Banach space $E$ and by $\langle\cdot, \cdot\rangle$ we denote the duality between $E$ and $E^{*}$. By $L^{s}(I ; E)$ we denote the Bochner space (see [1]). Therefore, $L^{s}(I ; E)^{*}=L^{s^{\prime}}\left(I ; E^{*}\right)$.

\subsection{Structure and data properties}

We start by introducing several structural assumptions on functions in $1.1-1.8$ :

(i) $S \in C^{1}(\mathbb{R})$ is a positive and strictly monotone function such that

$$
\begin{array}{ll}
0<S(\xi) \leq S_{s}<+\infty & \forall \xi \in \mathbb{R} \quad\left(S_{s}=\text { const }\right), \\
\left(S\left(\xi_{1}\right)-S\left(\xi_{2}\right)\right)\left(\xi_{1}-\xi_{2}\right)>0 & \forall \xi_{1}, \xi_{2} \in \mathbb{R}, \xi_{1} \neq \xi_{2} .
\end{array}
$$

(ii) $a, a_{1}, k, k_{R}, \mu, \varrho, D_{w}, \lambda$ are continuous functions, $a_{1}$ strictly increasing, satisfying

$$
\begin{array}{ll}
0<k_{1} \leq k(x, \xi) \leq k_{2}<+\infty \quad\left(k_{1}, k_{2}=\text { const }\right) & \forall \xi \in \mathbb{R}, x \in \Omega, \\
k_{R} \in C\left(\left[0, S_{s}\right]\right),\left(k_{R}\left(\xi_{1}\right)-k_{R}\left(\xi_{2}\right)\right)\left(\xi_{1}-\xi_{2}\right)>0 & \forall \xi_{1}, \xi_{2} \in\left[0, S_{s}\right], \\
& \xi_{1} \neq \xi_{2}, \\
0<k_{R}(\xi) & \forall \xi \in\left[0, S_{s}\right], \\
0<\mu_{1} \leq \mu(\xi) \leq \mu_{2}<+\infty \quad\left(\mu_{1}, \mu_{2}=\text { const }\right) & \forall \xi \in \mathbb{R}, \\
0<\varrho_{1} \leq \varrho(x, \xi) \leq \varrho_{2}<+\infty \quad\left(\varrho_{1}, \varrho_{2}=\text { const }\right) & \forall \xi \in \mathbb{R}, x \in \Omega, \quad(2.5) \\
0<a_{1}\left(\xi_{1}\right) \leq a\left(x, \xi_{1}, \xi_{2}, \xi_{3}\right) \leq a_{2}<+\infty & \forall \xi_{1}, \xi_{2}, \xi_{3} \in \mathbb{R}, \quad(2.8) \\
& x \in \Omega \quad\left(a_{2}=\text { const }\right), \\
0<D_{w}(x, \xi) & \forall \xi \in \mathbb{R}, x \in \Omega, \quad(2.9) \\
0<\lambda\left(x, \xi_{1}, \xi_{2}, \xi_{3}\right) & \forall \xi_{1}, \xi_{2}, \xi_{3} \in \mathbb{R}, \quad(2.10) \\
& x \in \Omega .
\end{array}
$$

(iii) The function $\phi$ is Lipschitz continuous with respect to the second variable, i.e. there exists a constant $C_{\phi}>0$ such that

$$
\left|\phi\left(x, \xi_{1}\right)-\phi\left(x, \xi_{2}\right)\right| \leq C_{\phi}\left|\xi_{1}-\xi_{2}\right| \quad \forall \xi_{1}, \xi_{2} \in \mathbb{R}, x \in \Omega
$$


and

$$
0<\phi_{1} \leq \phi(x, \xi) \leq \phi_{2}<+\infty \quad \forall \xi \in \mathbb{R}, x \in \Omega \quad\left(\phi_{1}, \phi_{2}=\text { const }\right)
$$

(iv) $f$ is Lipschitz continuous in all respective variables and there exists an increasing positive bounded function $\tilde{f}$ such that $\left(C_{f}=\right.$ const $)$

$$
\left|f\left(x, \xi_{1}, \xi_{2}, \xi_{3}, \xi_{4}\right)\right| \leq \tilde{f}\left(\xi_{1}\right) \leq C_{f} \quad \forall \xi_{1}, \xi_{2}, \xi_{3}, \xi_{4} \in \mathbb{R}, x \in \Omega
$$

(v) We assume that there exists a non-increasing positive function $M$ such that

$$
M(\xi) \leq \frac{a_{1}(\xi)}{S^{\prime}(\xi)} \quad \forall \xi \in \mathbb{R}
$$

and

$$
\lim _{\delta \rightarrow 0_{+}} \frac{\tilde{f}\left(S^{-1}(\delta)\right)}{M\left(S^{-1}(\delta)\right) \delta}=0 .
$$

(vi) (Initial data) Assume $c_{0}, \vartheta_{0} \in W^{1,2} \cap L^{\infty}(\Omega)$ and $p_{0} \in L^{\infty}(\Omega)$ such that

$$
-\infty<p_{1}<p_{0}(\cdot) \leq 0 \quad \text { a.e. in } \Omega \quad\left(p_{1}=\text { const }\right)
$$

Throughout the paper the hypotheses (i)-(vi) will be assumed.

\section{The main result}

The aim of this paper is to prove the existence of a weak solution to the problem 1.1 (1.8). We first reformulate the problem in a variational sense.

Definition 3.1 A weak solution of (1.1)-1.8 is a foursome $[p, c, \vartheta, r]$ such that

$$
\begin{aligned}
& p \in L^{2}\left(I ; W_{\Gamma_{D}}^{1,2}(\Omega)\right), \\
& c \in L^{2}\left(I ; W_{\Gamma_{D}}^{1,2}(\Omega)\right) \cap L^{\infty}\left(Q_{T}\right), \\
& \vartheta \in L^{2}\left(I ; W_{\Gamma_{D}}^{1,2}(\Omega)\right) \cap L^{\infty}\left(Q_{T}\right), \\
& r \in C\left([0, T] ; L^{\infty}(\Omega)\right)
\end{aligned}
$$

and

$$
\begin{aligned}
-\int_{Q_{T}} \phi(x, r) & S(p) \partial_{t} \zeta \mathrm{d} x \mathrm{~d} t+\int_{Q_{T}} a(x, p, \vartheta, r) \nabla p \cdot \nabla \zeta \mathrm{d} x \mathrm{~d} t \\
= & \int_{\Omega} \phi\left(x, r_{0}\right) S\left(p_{0}\right) \zeta(x, 0) \mathrm{d} x+\int_{Q_{T}} \alpha_{1} f(x, p, c, \vartheta, r) \zeta \mathrm{d} x \mathrm{~d} t
\end{aligned}
$$

for any $\zeta \in L^{2}\left(I ; W_{\Gamma_{D}}^{1,2}(\Omega)\right) \cap W^{1,1}\left(I ; L^{1}(\Omega)\right)$ with $\zeta(\cdot, T)=0$; 


$$
\begin{array}{r}
-\int_{Q_{T}} \phi(x, r) S(p) c \partial_{t} \eta \mathrm{d} x \mathrm{~d} t+\int_{Q_{T}} \phi(x, r) S(p) D_{w}(x, p) \nabla c \cdot \nabla \eta \mathrm{d} x \mathrm{~d} t \\
\quad+\int_{Q_{T}} c a(x, p, \vartheta, r) \nabla p \cdot \nabla \eta \mathrm{d} x \mathrm{~d} t=\int_{\Omega} \phi\left(x, r_{0}\right) S\left(p_{0}\right) c_{0} \eta(x, 0) \mathrm{d} x
\end{array}
$$

for any $\eta \in L^{2}\left(I ; W_{\Gamma_{D}}^{1,2}(\Omega)\right) \cap W^{1,1}\left(I ; L^{1}(\Omega)\right)$ with $\eta(\cdot, T)=0$;

$$
\begin{aligned}
& -\int_{Q_{T}}[\phi(x, r) S(p)+\varrho(x, r)] \vartheta \partial_{t} \psi \mathrm{d} x \mathrm{~d} t \\
& \int_{Q_{T}} \lambda(x, p, \vartheta, r) \nabla \vartheta \cdot \nabla \psi \mathrm{d} x \mathrm{~d} t+\int_{Q_{T}} \vartheta a(x, p, \vartheta, r) \nabla p \cdot \nabla \psi \mathrm{d} x \mathrm{~d} t \\
= & \int_{\Omega}\left[\phi\left(x, r_{0}\right) S\left(p_{0}\right)+\varrho\left(x, r_{0}\right)\right] \vartheta_{0} \psi(x, 0) \mathrm{d} x+\int_{Q_{T}} \alpha_{2} f(x, p, c, \vartheta, r) \psi \mathrm{d} x \mathrm{~d} t
\end{aligned}
$$

for any $\psi \in L^{2}\left(I ; W_{\Gamma_{D}}^{1,2}(\Omega)\right) \cap W^{1,1}\left(I ; L^{1}(\Omega)\right)$ with $\psi(\cdot, T)=0$, where

$$
r(t)=\int_{0}^{t} f(x, p(x, s), c(x, s), \vartheta(x, s), r(x, s)) \mathrm{d} s \quad \text { in } L^{\infty}(\Omega) \text { for all } t \in[0, T] .
$$

The main result of this paper reads as follows:

Theorem 3.2 (Main result) Let the assumptions (i)-(vi) be satisfied. Then there exists at least one weak solution of the system (1.1)-1.8).

To prove the main result of the paper we use the method of semidiscretization in time by constructing temporal approximations and limiting procedure. The proof can be divided into three steps. In the first step, we approximate our problem by means of a semi-implicit time discretization scheme (which preserve the pseudo-monotone structure of the discrete problem) and prove the existence and $W^{1, s}(\Omega)$-regularity (with some $s>2$ ) of discrete approximations. In the second step we derive necessary apriori estimates. The key point is to establish $L^{\infty}$-estimates to overcome degeneracies in transport coefficients. Finally, in the third step we construct temporal interpolants and pass to the limit from discrete approximations.

\section{Proof of the main result}

\subsection{Approximations}

Applying the method of discretization in time, we divide the interval $[0, T]$ into $n$ subintervals of lengths $h:=T / n$ (a time step), replace the time derivatives by the corresponding difference quotients and the integral in (3.4) by a sum. In this way, we approximate the problem (1.1)-(1.8) by a semi-implicit time discretization scheme and re-formulate the problem in a weak sense. 
Let us consider $p_{n}^{0}:=p_{0}, c_{n}^{0}:=c_{0}, \vartheta_{n}^{0}:=\vartheta_{0}$ and $r_{n}^{0}:=0$ a.e. on $\Omega$. We now define, in each time step $i=1, \ldots, n$, a foursome $\left[p_{n}^{i}, c_{n}^{i}, \vartheta_{n}^{i}, r_{n}^{i}\right]$ as a solution of the following recurrence steady problem: for a given foursome $\left[p_{n}^{i-1}, c_{n}^{i-1}, \vartheta_{n}^{i-1}, r_{n}^{i-1}\right]$, $i=1,2, \ldots, n, p_{n}^{i-1} \in L^{\infty}(\Omega), c_{n}^{i-1} \in W^{1,2}(\Omega) \cap L^{\infty}(\Omega), \vartheta_{n}^{i-1} \in W^{1,2}(\Omega) \cap L^{\infty}(\Omega)$ and $r_{n}^{i-1} \in W^{1,2}(\Omega) \cap L^{\infty}(\Omega)$, find $\left[p_{n}^{i}, c_{n}^{i}, \vartheta_{n}^{i}, r_{n}^{i}\right]$, such that $p_{n}^{i} \in W_{\Gamma_{D}}^{1,2}(\Omega) \cap L^{\infty}(\Omega)$, $c_{n}^{i} \in W_{\Gamma_{D}}^{1,2}(\Omega) \cap L^{\infty}(\Omega), \vartheta_{n}^{i} \in W_{\Gamma_{D}}^{1,2}(\Omega) \cap L^{\infty}(\Omega), r_{n}^{i} \in W^{1,2}(\Omega) \cap L^{\infty}(\Omega)$ and

$$
\begin{aligned}
& \int_{\Omega} \frac{\phi\left(x, r_{n}^{i}\right) S\left(p_{n}^{i}\right)-\phi\left(x, r_{n}^{i-1}\right) S\left(p_{n}^{i-1}\right)}{h} \zeta \mathrm{d} x \\
& +\int_{\Omega} a\left(x, p_{n}^{i}, \vartheta_{n}^{i-1}, r_{n}^{i-1}\right) \nabla p_{n}^{i} \cdot \nabla \zeta \mathrm{d} x \\
= & \int_{\Omega} \alpha_{1} f\left(x, p_{n}^{i}, c_{n}^{i-1}, \vartheta_{n}^{i-1}, r_{n}^{i-1}\right) \zeta \mathrm{d} x
\end{aligned}
$$

for any $\zeta \in W_{\Gamma_{D}}^{1,2}(\Omega)$;

$$
\begin{aligned}
& \int_{\Omega} \frac{\phi\left(x, r_{n}^{i}\right) S\left(p_{n}^{i}\right) c_{n}^{i}-\phi\left(x, r_{n}^{i-1}\right) S\left(p_{n}^{i-1}\right) c_{n}^{i-1}}{h} \eta \mathrm{d} x \\
& +\int_{\Omega} \phi\left(x, r_{n}^{i}\right) S\left(p_{n}^{i}\right) D_{w}\left(x, p_{n}^{i}\right) \nabla c_{n}^{i} \cdot \nabla \eta \mathrm{d} x \\
& +\int_{\Omega} c_{n}^{i} a\left(x, p_{n}^{i}, \vartheta_{n}^{i-1}, r_{n}^{i-1}\right) \nabla p_{n}^{i} \cdot \nabla \eta \mathrm{d} x \\
& =0
\end{aligned}
$$

for any $\eta \in W_{\Gamma_{D}}^{1,2}(\Omega)$;

$$
\begin{aligned}
& \quad \int_{\Omega} \frac{\phi\left(x, r_{n}^{i}\right) S\left(p_{n}^{i}\right) \vartheta_{n}^{i}-\phi\left(x, r_{n}^{i-1}\right) S\left(p_{n}^{i-1}\right) \vartheta_{n}^{i-1}}{h} \psi \mathrm{d} x \\
& +\int_{\Omega} \frac{\varrho\left(x, r_{n}^{i}\right) \vartheta_{n}^{i}-\varrho\left(x, r_{n}^{i-1}\right) \vartheta_{n}^{i-1}}{h} \psi \mathrm{d} x \\
& +\int_{\Omega} \lambda\left(x, p_{n}^{i-1}, \vartheta_{n}^{i-1}, r_{n}^{i-1}\right) \nabla \vartheta_{n}^{i} \cdot \nabla \psi \mathrm{d} x \\
& +\int_{\Omega} \vartheta_{n}^{i} a\left(x, p_{n}^{i}, \vartheta_{n}^{i-1}, r_{n}^{i-1}\right) \nabla p_{n}^{i} \cdot \nabla \psi \mathrm{d} x \\
& =\int_{\Omega} \alpha_{2} f\left(x, p_{n}^{i}, c_{n}^{i-1}, \vartheta_{n}^{i-1}, r_{n}^{i-1}\right) \psi \mathrm{d} x
\end{aligned}
$$

for any $\psi \in W_{\Gamma_{D}}^{1,2}(\Omega)$ and

$$
\begin{aligned}
& r_{n}^{i}=h \sum_{j=1}^{i} f\left(x, p_{n}^{j}, c_{n}^{j-1}, \vartheta_{n}^{j-1}, r_{n}^{j-1}\right), \quad i=1, \ldots, n, \\
& r_{n}^{0}(x)=0 .
\end{aligned}
$$


Theorem 4.1 (Existence of the solution to 4.1)-4.4) Let $p_{n}^{i-1} \in L^{\infty}(\Omega), c_{n}^{i-1} \in$ $W^{1,2}(\Omega) \cap L^{\infty}(\Omega), \vartheta_{n}^{i-1} \in W^{1,2}(\Omega) \cap L^{\infty}(\Omega)$ and $r_{n}^{i-1} \in W^{1,2}(\Omega) \cap L^{\infty}(\Omega)$ be given and the Assumptions (i)-(vi) be satisfied. Then there exists $\left[p_{n}^{i}, c_{n}^{i}, \vartheta_{n}^{i}, r_{n}^{i}\right]$, such that $p_{n}^{i} \in W_{\Gamma_{D}}^{1, s}(\Omega), c_{n}^{i} \in W_{\Gamma_{D}}^{1, s}(\Omega), \vartheta_{n}^{i} \in W_{\Gamma_{D}}^{1, s}(\Omega)$ with some $s>2$, and $r_{n}^{i} \in$ $W^{1,2}(\Omega) \cap L^{\infty}(\Omega)$, satisfying (4.1)-(4.4).

Remark 4.2 By Theorem 4.1 and the embeddings $W_{\Gamma_{D}}^{1, s}(\Omega) \hookrightarrow L^{\infty}(\Omega)$ (recall that $s>2$ and $\Omega \subset \mathbb{R}^{2}$ ) and $W_{\Gamma_{D}}^{1, s}(\Omega) \hookrightarrow W^{1,2}(\Omega)$ we are able to solve (4.1)-(4.4) recursively for $\left[p_{n}^{i}, c_{n}^{i}, \vartheta_{n}^{i}, r_{n}^{i}\right]$ by the already known $\left[p_{n}^{i-1}, c_{n}^{i-1}, \vartheta_{n}^{i-1}, r_{n}^{i-1}\right]$, such that we obtain

$$
\begin{aligned}
& p_{n}^{i} \in W_{\Gamma_{D}}^{1,2}(\Omega) \cap L^{\infty}(\Omega), \\
& c_{n}^{i} \in W_{\Gamma_{D}}^{1,2}(\Omega) \cap L^{\infty}(\Omega), \\
& \vartheta_{n}^{i} \in W_{\Gamma_{D}}^{1,2}(\Omega) \cap L^{\infty}(\Omega), \\
& r_{n}^{i} \in W^{1,2}(\Omega) \cap L^{\infty}(\Omega)
\end{aligned}
$$

for all $i=1, \ldots, n$.

Before proving Theorem 4.1, we present two auxiliary results, formulated in Theorem 4.3 and Lemma 4.4 .

Theorem 4.3 (Weak maximum principle for pressure approximations) $\operatorname{Let} p_{n}^{i} \in W_{\Gamma_{D}}^{1, s}(\Omega)$, $c_{n}^{i} \in W_{\Gamma_{D}}^{1, s}(\Omega), \vartheta_{n}^{i} \in W_{\Gamma_{D}}^{1, s}(\Omega)$ with some $s>2$, and $r_{n}^{i} \in W^{1,2}(\Omega) \cap L^{\infty}(\Omega)$ solve (4.1)-(4.5) successively for $i=1, \ldots, n$. Then there exists $\ell$ (independent of $n$ ) such that

$$
p_{n}^{i} \geq \ell \text { almost everywhere in } \Omega \text { and for all } i=1,2, \ldots, n .
$$

To prove Theorem 4.3 we need the following lemma:

Lemma 4.4 (See e.g. Proposition 4.2 in [36]) If a nonnegative sequence $\left\{Z_{j}\right\}$ satisfies

$$
Z_{j+1} \leq \gamma 4^{j} Z_{j}^{\tau+1} \quad(\tau>0)
$$

then

$$
\lim _{j \rightarrow+\infty} Z_{j}=0
$$

provided that

$$
\gamma \leq Z_{0}^{-\tau} 4^{-1 / \tau}
$$

Proof. The proof follows from the proof of Lemma 4.1.1 in [49].

Proof of Theorem 4.3 The proof is based on the De Giorgi iteration technique, see e.g. [49, Chapter 4] or [27]. Let $k \in \mathbb{R}$ and set

$$
(\phi-k)_{-} \equiv \begin{cases}\phi-k, & \phi<k \\ 0, & \phi \geq k\end{cases}
$$


For $k<p_{1}$ (here, $p_{1}$ is taken from (2.16) $)$ we have $\zeta=\left(S\left(p_{n}^{i}\right)-S(k)\right)_{-} \in W_{\Gamma_{D}}^{1,2}(\Omega)$ and thus we may choose $\zeta=\left(S\left(p_{n}^{i}\right)-S(k)\right)_{-}$as a test function in (4.1). It is a matter of a simple technical computation to arrive at the estimate

$$
\begin{aligned}
& \frac{1}{2 h} \int_{\Omega} \phi\left(x, r_{n}^{i}\right)\left|\left(S\left(p_{n}^{i}\right)-S(k)\right)_{-}\right|^{2} \mathrm{~d} x \\
& -\frac{1}{2 h} \int_{\Omega} \phi\left(x, r_{n}^{i-1}\right)\left|\left(S\left(p_{n}^{i-1}\right)-S(k)\right)_{-}\right|^{2} \mathrm{~d} x \\
& +\int_{\Omega} a\left(x, p_{n}^{i}, \vartheta_{n}^{i-1}, r_{n}^{i-1}\right) \frac{1}{S^{\prime}\left(p_{n}^{i}\right)}\left|\nabla\left(S\left(p_{n}^{i}\right)-S(k)\right)_{-}\right|^{2} \mathrm{~d} x \\
\leq & \int_{\Omega} \alpha_{1} f\left(x, p_{n}^{i}, c_{n}^{i-1}, \vartheta_{n}^{i-1}, r_{n}^{i-1}\right)\left(S\left(p_{n}^{i}\right)-S(k)\right)_{-} \mathrm{d} x \\
& -\frac{1}{2 h} \int_{\Omega}\left[\phi\left(x, r_{n}^{i}\right)-\phi\left(x, r_{n}^{i-1}\right)\right]\left(S\left(p_{n}^{i}\right)+S(k)\right)\left(S\left(p_{n}^{i}\right)-S(k)\right)_{-} \mathrm{d} x .
\end{aligned}
$$

Using the Lipschitz continuity of $\phi$ with respect to $r$, see (2.11), and using (4.4), we can write

$$
\begin{aligned}
\left|\phi\left(x, r_{n}^{i}\right)-\phi\left(x, r_{n}^{i-1}\right)\right| & \leq C_{\phi}\left|r_{n}^{i}-r_{n}^{i-1}\right| \\
& \leq h C_{\phi}\left|f\left(x, p_{n}^{i}, c_{n}^{i-1}, \vartheta_{n}^{i-1}, r_{n}^{i-1}\right)\right| .
\end{aligned}
$$

Let us denote

$$
I_{k}(i):=\int_{\Omega} \phi\left(x, r_{n}^{i}\right)\left|\left(S\left(p_{n}^{i}\right)-S(k)\right)_{-}\right|^{2} \mathrm{~d} x, \quad i=1, \ldots, n
$$

and let $I_{k}$ attains its maximum at $i=m$, i.e.

$$
I_{k}(m)=\max _{i=1, \ldots, n} I_{k}(i)
$$

and, in other words,

$$
I_{k}(m) \geq I_{k}(i) \quad \text { for all } i=1,2, \ldots, n .
$$

From this and in view of (4.8) and (4.9) we have

$$
\begin{aligned}
& \int_{\Omega} a\left(x, p_{n}^{m}, \vartheta_{n}^{m-1}, r_{n}^{m-1}\right) \frac{1}{S^{\prime}\left(p_{n}^{m}\right)}\left|\nabla\left(S\left(p_{n}^{m}\right)-S(k)\right)_{-}\right|^{2} \mathrm{~d} x \\
\leq & \left(C_{\phi} S_{s}+\left|\alpha_{1}\right|\right) \int_{\Omega}\left|f\left(x, p_{n}^{m}, c_{n}^{m-1}, \vartheta_{n}^{m-1}, r_{n}^{m-1}\right)\left(S\left(p_{n}^{m}\right)-S(k)\right)_{-}\right| \mathrm{d} x .
\end{aligned}
$$

Further, using (2.8) leads to

$$
\begin{aligned}
& \int_{\Omega} \frac{a_{1}\left(p_{n}^{m}\right)}{S^{\prime}\left(p_{n}^{m}\right)}\left|\nabla\left(S\left(p_{n}^{m}\right)-S(k)\right)_{-}\right|^{2} \mathrm{~d} x \\
\leq & \left(C_{\phi} S_{s}+\left|\alpha_{1}\right|\right) \int_{\Omega}\left|f\left(x, p_{n}^{m}, c_{n}^{m-1}, \vartheta_{n}^{m-1}, r_{n}^{m-1}\right)\left(S\left(p_{n}^{m}\right)-S(k)\right)_{-}\right| \mathrm{d} x .
\end{aligned}
$$


On the other hand, by Assumption (v), namely, the inequality (2.14), we see that

$$
M(k) \int_{\Omega}\left|\nabla\left(S\left(p_{n}^{m}\right)-S(k)\right)_{-}\right|^{2} \mathrm{~d} x \leq \int_{\Omega} \frac{a\left(p_{n}^{m}\right)}{S^{\prime}\left(p_{n}^{m}\right)}\left|\nabla\left(S\left(p_{n}^{m}\right)-S(k)\right)_{-}\right|^{2} \mathrm{~d} x .
$$

Using the embedding theorem gives (recall that $\Omega$ is a two-dimensional domain)

$$
\begin{aligned}
M(k)\left(\int_{\Omega}\left|\left(S\left(p_{n}^{m}\right)-S(k)\right)_{-}\right|^{q} \mathrm{~d} x\right)^{2 / q} & \\
& \leq C_{E} M(k) \int_{\Omega}\left|\nabla\left(S\left(p_{n}^{m}\right)-S(k)\right)_{-}\right|^{2} \mathrm{~d} x,
\end{aligned}
$$

where $2<q<+\infty$ and, here, the embedding constant $C_{E}$ depends only on $\Omega$. We may now combine (4.14)-(4.16) to obtain

$$
\begin{aligned}
& M(k)\left(\int_{\Omega}\left|\left(S\left(p_{n}^{m}\right)-S(k)\right)_{-}\right|^{q} \mathrm{~d} x\right)^{2 / q} \\
& \quad \leq C_{E}\left(C_{\phi} S_{s}+\left|\alpha_{1}\right|\right) \int_{\Omega}\left|f\left(x, p_{n}^{m}, c_{n}^{m-1}, \vartheta_{n}^{m-1}, r_{n}^{m-1}\right)\left(S\left(p_{n}^{m}\right)-S(k)\right)_{-}\right| \mathrm{d} x .
\end{aligned}
$$

Taking into account (2.13) we have

$$
\begin{aligned}
& \int_{\Omega} \mid f\left(x, p_{n}^{m}, c_{n}^{m-1}, \vartheta_{n}^{m-1}, r_{n}^{m-1}\right)\left(S\left(p_{n}^{m}\right)\right.-S(k))_{-} \mid \mathrm{d} x \\
& \leq \tilde{f}(k) \int_{\Omega}\left|\left(S\left(p_{n}^{m}\right)-S(k)\right)_{-}\right| \mathrm{d} x
\end{aligned}
$$

and applying the Hölder's inequality to the right hand side in (4.18) and combining (4.17) and (4.18) we arrive at the estimate

$$
\begin{aligned}
\left(\int_{A_{k}(m)}\left|\left(S\left(p_{n}^{m}\right)-S(k)\right)_{-}\right|^{q} \mathrm{~d} x\right)^{1 / q} & \\
& \leq \frac{C_{E}\left(C_{\phi} S_{s}+\left|\alpha_{1}\right|\right) \tilde{f}(k)}{M(k)}\left(\int_{A_{k}(m)} 1 \mathrm{~d} x\right)^{1 / q^{\prime}},
\end{aligned}
$$

where

$$
A_{k}(m)=\left\{x \in \Omega ; p_{n}^{m}(x)<k\right\} .
$$

On the other hand, applying the Hölder's inequality we have

$$
\begin{aligned}
& \int_{A_{k}(m)}\left|\left(S\left(p_{n}^{m}\right)-S(k)\right)_{-}\right|^{2} \mathrm{~d} x \\
\leq & \left(\int_{A_{k}(m)}\left|\left(S\left(p_{n}^{m}\right)-S(k)\right)_{-}\right|^{q} \mathrm{~d} x\right)^{2 / q}\left(\int_{A_{k}(m)} 1 \mathrm{~d} x\right)^{(q-2) / q}
\end{aligned}
$$


and using (4.19) yields

$$
\begin{aligned}
& \int_{A_{k}(m)}\left|\left(S\left(p_{n}^{m}\right)-S(k)\right)_{-}\right|^{2} \mathrm{~d} x \\
\leq & \left(\frac{C_{E}\left(C_{\phi} S_{s}+\left|\alpha_{1}\right|\right) \tilde{f}(k)}{M(k)}\right)^{2}\left(\int_{A_{k}(m)} 1 \mathrm{~d} x\right)^{(3 q-4) / q} .
\end{aligned}
$$

In view of (4.11) and employing (2.12) we can write

$$
\begin{aligned}
\phi_{1} \int_{A_{k}(i)}\left|\left(S\left(p_{n}^{i}\right)-S(k)\right)_{-}\right|^{2} \mathrm{~d} x & \leq I_{k}(i) \\
& \leq I_{k}(m) \leq \phi_{2} \int_{A_{k}(m)}\left|\left(S\left(p_{n}^{m}\right)-S(k)\right)_{-}\right|^{2} \mathrm{~d} x
\end{aligned}
$$

for all $i=1,2, \ldots, n$. Since $\ell<k$ implies $A_{\ell}(i) \subset A_{k}(i)$, and $\left(S\left(p_{n}^{i}\right)-S(k)\right)_{-} \leq$ $(S(\ell)-S(k))<0$ on $A_{\ell}(i)$, we have

$$
\mid S(\ell)-S(k))\left.\right|^{2} \leq\left|\left(S\left(p_{n}^{i}\right)-S(k)\right)_{-}\right|^{2} \quad \text { on } A_{\ell}(i)
$$

and thus

$$
\begin{aligned}
(S(\ell)-S(k))^{2}\left|A_{\ell}(i)\right| & \leq \int_{A_{\ell}(i)}\left|\left(S\left(p_{n}^{i}\right)-S(k)\right)_{-}\right|^{2} \mathrm{~d} x \\
& \leq \int_{A_{k}(i)}\left|\left(S\left(p_{n}^{i}\right)-S(k)\right)_{-}\right|^{2} \mathrm{~d} x \\
& \leq \frac{\phi_{2}}{\phi_{1}} \int_{A_{k}(m)}\left|\left(S\left(p_{n}^{m}\right)-S(k)\right)_{-}\right|^{2} \mathrm{~d} x .
\end{aligned}
$$

Finally, from this and (4.20) we deduce

$$
\left|A_{\ell}(i)\right| \leq \frac{\phi_{2}}{\phi_{1}}\left(\frac{C_{E}\left(C_{\phi} S_{s}+\left|\alpha_{1}\right|\right) \tilde{f}(k)}{M(k)(S(\ell)-S(k))}\right)^{2}\left|A_{k}(m)\right|^{(3 q-4) / q} \quad \text { for all } i=1, \ldots, n .
$$

To conclude the proof of Theorem 4.3, we define

$$
\mu_{k}=\max _{i=1, \ldots, n}\left|A_{k}(i)\right| .
$$

Now, (4.23) implies (recall $\ell<k$ )

$$
\left|\mu_{\ell}\right| \leq \frac{\phi_{2}}{\phi_{1}}\left(\frac{C_{E}\left(C_{\phi} S_{s}+\left|\alpha_{1}\right|\right) \tilde{f}(k)}{M(k)(S(\ell)-S(k))}\right)^{2}\left|\mu_{k}\right|^{(3 q-4) / q} .
$$

Next, we are going to apply Lemma4.4 In particular, we define a decreasing sequence

$$
d_{j}=\frac{\delta}{2}\left(1+\frac{1}{2^{j}}\right), \quad j=0,1,2, \ldots,
$$


where $\delta$ is a small positive real number and let

$$
k_{j}=S^{-1}\left(d_{j}\right) .
$$

Since $S$ is strictly increasing function, it is clear that

$$
k_{j+1}<k_{j}, \quad j=0,1,2, \ldots
$$

Then from (4.24) we have

$$
\begin{aligned}
Z_{j+1} & \leq \frac{\phi_{2}}{\phi_{1}}\left(\frac{C_{E}\left(C_{\phi} S_{s}+\left|\alpha_{1}\right|\right) \tilde{f}\left(S^{-1}\left(d_{j}\right)\right)}{M\left(S^{-1}\left(d_{j}\right)\right)\left(d_{j+1}-d_{j}\right)}\right)^{2} Z_{j}^{(3 q-4) / q} \\
& \leq \frac{\phi_{2}}{\phi_{1}}\left(\frac{C_{E}\left(C_{\phi} S_{s}+\left|\alpha_{1}\right|\right) \tilde{f}\left(S^{-1}(\delta)\right) 4}{M\left(S^{-1}(\delta)\right) \delta}\right)^{2} 4^{j} Z_{j}^{[1+2(q-2) / q]},
\end{aligned}
$$

where

$$
Z_{j}=\left|\mu_{k_{j}}\right| .
$$

Recall that $2<q<+\infty$. In view of Assumption (v) and taking $\delta>0$ "small enough" we apply Lemma 4.4 to get (4.7). Explicitly, this means that there exists $\ell$ (independent of $n$ ) such that

$$
\left|\mu_{\ell}\right|=0
$$

in other words,

$$
p_{n}^{i} \geq \ell \text { almost everywhere in } \Omega \text { and for all } i=1, \ldots, n .
$$

The proof of Theorem 4.3 is complete.

Now we are ready to prove Theorem 4.1

Proof of Theorem 4.1 The proof rests on the $W^{1, s}$-regularity of elliptic problems presented in [12, 20] and the embedding $W_{\Gamma_{D}}^{1, s}(\Omega) \subset L^{\infty}(\Omega)$ if $s>2$ (recall that $\Omega$ is a bounded domain in $\mathbb{R}^{2}$ ).

We begin by proving the existence of $p_{n}^{i} \in W_{\Gamma_{D}}^{1,2}(\Omega)$, being the solution to problem (4.1). Due to Theorem 4.3, we may consider the truncated function $\widetilde{k}_{r}$ defined by

$$
\widetilde{k}_{r}(\xi) \equiv \begin{cases}k_{r}(S(\xi)), & \xi>\ell \\ k_{r}(S(\ell)), & \xi \leq \ell\end{cases}
$$

where $\ell$ is taken from (4.6). Recall that $k_{R}$ is positive and strictly increasing on $\left[0, S_{s}\right]$ and $S$ is positive and strictly increasing on $\mathbb{R}$. Hence $\widetilde{k}_{r}$ is the increasing function such that

$$
0<K_{0} \leq \widetilde{k}_{r}(\xi) \leq K_{1} \quad \forall \xi \in \mathbb{R}
$$


with appropriate chosen constants $K_{0}$ and $K_{1}$, say $K_{0}=k_{r}(S(\ell))$ and $K_{1}=k_{r}\left(S_{s}\right)$. Hence, problem (4.1) takes the form

$$
\begin{aligned}
& \int_{\Omega} \frac{k\left(x, r_{n}^{i-1}(x)\right)}{\mu\left(\vartheta_{n}^{i-1}(x)\right)} \widetilde{k}_{r}\left(p_{n}^{i}\right) \nabla p_{n}^{i} \cdot \nabla \zeta \mathrm{d} x \\
& +\frac{1}{h} \int_{\Omega} \phi\left(x, r_{n}^{i}\right) S\left(p_{n}^{i}\right) \zeta \mathrm{d} x-\int_{\Omega} \alpha_{1} f\left(x, p_{n}^{i}, c_{n}^{i-1}, \vartheta_{n}^{i-1}, r_{n}^{i-1}\right) \zeta \mathrm{d} x \\
= & \frac{1}{h} \int_{\Omega} \phi\left(x, r_{n}^{i-1}\right) S\left(p_{n}^{i-1}\right) \zeta \mathrm{d} x
\end{aligned}
$$

for any $\zeta \in W_{\Gamma_{D}}^{1,2}(\Omega)$. Note that the unknown $r_{n}^{i}$ in the second line of (4.28) can be easily eliminated using the equation (4.4), which can be rewritten as

$$
r_{n}^{i}=r_{n}^{i-1}+h f\left(x, p_{n}^{i}, c_{n}^{i-1}, \vartheta_{n}^{i-1}, r_{n}^{i-1}\right) .
$$

We now define the so called Kirchhoff transformation, which employs the primitive function $\beta: \mathbb{R} \rightarrow \mathbb{R}, \zeta=\beta(\xi)$, defined by

$$
\beta(\xi)=\int_{0}^{\xi} \widetilde{k}_{r}(s) \mathrm{d} s .
$$

It is worth noting that (4.27) implies $\beta$ to be continuous and increasing, and one-to-one with $\beta^{-1}$ Lipschitz continuous. Hence, with the notation $u(x)=\beta\left(p_{n}^{i}(x)\right)$, problem (4.28) can be rewritten in terms of a new variable $u$ as

$$
\int_{\Omega} A(x) \nabla u \cdot \nabla \zeta \mathrm{d} x+\int_{\Omega} B(x, u) \zeta \mathrm{d} x=\int_{\Omega} g(x) \zeta \mathrm{d} x
$$

for any $\zeta \in W_{\Gamma_{D}}^{1,2}(\Omega)$, where we denote briefly

$$
\begin{aligned}
A(x)= & \frac{k\left(x, r_{n}^{i-1}(x)\right)}{\mu\left(\vartheta_{n}^{i-1}(x)\right)}, \\
B(x, u)= & \frac{S\left(\beta^{-1}(u)\right)}{h} \phi\left(x, r_{n}^{i-1}(x)+h f\left(x, \beta^{-1}(u), c_{n}^{i-1}(x), \vartheta_{n}^{i-1}(x), r_{n}^{i-1}(x)\right)\right) \\
& -\alpha_{1} f\left(x, \beta^{-1}(u), c_{n}^{i-1}(x), \vartheta_{n}^{i-1}(x), r_{n}^{i-1}(x)\right), \\
g(x)= & \frac{\phi\left(x, r_{n}^{i-1}(x)\right) S\left(p_{n}^{i-1}\right)}{h} .
\end{aligned}
$$

Note that $g \in L^{\infty}(\Omega)$ and

$$
\begin{aligned}
& 0<A_{1}<A(\cdot)<A_{2}<+\infty \quad\left(A_{1}, A_{2}=\text { const }\right) \quad \text { a.e. in } \Omega, \\
& |B(\cdot, \xi)| \leq C \quad \forall \xi \in \mathbb{R} \text { and a.e. in } \Omega \text {. }
\end{aligned}
$$

The existence of $u \in W_{\Gamma_{D}}^{1,2}(\Omega)$, the solution of problem [4.30), follows from [45, Chapter 2.4]. With $u \in W_{\Gamma_{D}}^{1,2}(\Omega)$ in hand, the weak maximum principle for the problem

$$
\int_{\Omega} A(x) \nabla u \cdot \nabla \zeta \mathrm{d} x=\int_{\Omega} g(x) \zeta \mathrm{d} x-\int_{\Omega} B(x, u) \zeta \mathrm{d} x
$$


for any $\zeta \in W_{\Gamma_{D}}^{1,2}(\Omega)$, gives the regularity $u \in L^{\infty}(\Omega)$, see e.g. [49, Chapter 4.1.2].

Note that, in view of (4.27), the Kirchhoff transformation preserves $L^{\infty}$ space for the problem. We now set $p_{n}^{i}(x):=\beta^{-1}(u(x))$ a.e. in $\Omega$ to get the representation

$$
\nabla p_{n}^{i}=\frac{1}{\widetilde{k}_{r}\left(\beta^{-1}(u)\right)} \nabla u, \quad \text { i.e. } \quad \widetilde{k}_{r}\left(p_{n}^{i}\right) \nabla p_{n}^{i}=\nabla u
$$

and hence

$$
p_{n}^{i} \in W_{\Gamma_{D}}^{1,2}(\Omega) \cap L^{\infty}(\Omega) \quad \text { iff } \quad u \in W_{\Gamma_{D}}^{1,2}(\Omega) \cap L^{\infty}(\Omega) .
$$

We now conclude that $p_{n}^{i}$ solves (4.1).

With $p_{n}^{i} \in W_{\Gamma_{D}}^{1,2}(\Omega) \cap L^{\infty}(\Omega)$ in hand, we rewrite the equation (4.1) in the form (transferring the lower-order terms to the right hand side)

$$
\begin{aligned}
& \int_{\Omega} a\left(x, p_{n}^{i}, \vartheta_{n}^{i-1}, r_{n}^{i-1}\right) \nabla p_{n}^{i} \cdot \nabla \zeta \mathrm{d} x \\
= & \int_{\Omega} \alpha_{1} f\left(x, p_{n}^{i}, c_{n}^{i-1}, \vartheta_{n}^{i-1}, r_{n}^{i-1}\right) \zeta \mathrm{d} x-\int_{\Omega} \frac{\phi\left(x, r_{n}^{i}\right) S\left(p_{n}^{i}\right)-\phi\left(x, r_{n}^{i-1}\right) S\left(p_{n}^{i-1}\right)}{h} \zeta \mathrm{d} x
\end{aligned}
$$

for any $\zeta \in W_{\Gamma_{D}}^{1,2}(\Omega)$. In view of Assumptions (i), (iii) and (iv), both integrals on the right hand side make sense for any $\zeta \in W_{\Gamma_{D}}^{1, r^{\prime}}(\Omega), r^{\prime}=r /(r-1)$ with some $r>2$. Now we are able to apply [12, Theorem 4] to obtain $p_{n}^{i} \in W_{\Gamma_{D}}^{1, s}(\Omega)$ with some $s>2$.

Now with $c_{n}^{i-1} \in W^{1,2}(\Omega), \vartheta_{n}^{i-1} \in W^{1,2}(\Omega), r_{n}^{i-1} \in W^{1,2}(\Omega)$ and $p_{n}^{i} \in W_{\Gamma_{D}}^{1, s}(\Omega)$ (with some $s>2$ ) in hand, one obtains $r_{n}^{i}$ directly from (4.29). Since $f$ is supposed to be Lipschitz continuous, we easily deduce $r_{n}^{i} \in W^{1,2}(\Omega)$ (c.f. [45, Proposition 1.28]). Moreover, by (2.13) we have $r_{n}^{i} \in L^{\infty}(\Omega)$.

The existence of $c_{n}^{i} \in W_{\Gamma_{D}}^{1,2}(\Omega)$ and $\vartheta_{n}^{i} \in W_{\Gamma_{D}}^{1,2}(\Omega)$, being the solutions to problems (4.2) and (4.3), respectively, can be proven in the same way as [4, Theorem 6.5]. In particular, with $p_{n}^{i} \in W_{\Gamma_{D}}^{1, s}(\Omega), s>2$, and $r_{n}^{i} \in L^{\infty}(\Omega)$, given by (4.4), in hand, (4.2) and (4.3) represent semilinear equations which can be solved by the approach in [45. Chapter 2.4]. Analysis similar to the above yields $c_{n}^{i}, \vartheta_{n}^{i} \in W_{\Gamma_{D}}^{1, s}(\Omega)$ with some $s>2$. By embedding theorem we have $c_{n}^{i}, \vartheta_{n}^{i} \in L^{\infty}(\Omega)$. The proof of Theorem 4.1 is complete.

\subsection{A-priori estimates for discrete approximations}

In this part of the paper, which is rather technical, we prove some uniform estimates (with respect to $n$ ) for the discrete approximations of the solution. In the following estimates, many different constants will appear. For simplicity of notation, as above, $C$, $C_{1}, C_{2}, \ldots$, represent generic constants which may change their numerical values from one formula to another but do not depend on $n$ and the functions under consideration. 


\subsubsection{Uniform bounds in $L^{\infty}$}

We first prove the apriori $L^{\infty}$-estimate for $c_{n}^{i}, i=1, \ldots, n$, being the solution to (4.2).

By Theorem 4.1 we have $c_{n}^{i} \in W_{\Gamma_{D}}^{1,2}(\Omega) \cap L^{\infty}(\Omega)$. Hence, $\left(c_{n}^{i}\right)^{\ell} \in W_{\Gamma_{D}}^{1,2}(\Omega) \cap L^{\infty}(\Omega)$ for all $\ell=1,2, \ldots$. The following procedure is similar to that used e.g. in [11, 41] for scalar problems. Let $\ell$ be an odd integer. Using $\zeta=[\ell /(\ell+1)]\left(c_{n}^{i}\right)^{\ell+1}$ as a test function in (4.1) and $\eta=\left(c_{n}^{i}\right)^{\ell}$ in (4.2) and combining both equations we obtain

$$
\begin{aligned}
& \frac{1}{h} \frac{1}{\ell+1} \int_{\Omega} \phi\left(x, r_{n}^{i}\right) S\left(p_{n}^{i}\right)\left[c_{n}^{i}\right]^{\ell+1} \mathrm{~d} x \\
& -\frac{1}{h} \frac{1}{\ell+1} \int_{\Omega} \phi\left(x, r_{n}^{i-1}\right) S\left(p_{n}^{i-1}\right)\left[c_{n}^{i-1}\right]^{\ell+1} \mathrm{~d} x \\
& +\frac{1}{h} \frac{1}{\ell+1} \int_{\Omega} \phi\left(x, r_{n}^{i-1}\right) S\left(p_{n}^{i-1}\right)\left[c_{n}^{i-1}\right]^{\ell+1} \mathrm{~d} x \\
& +\frac{1}{h} \frac{\ell}{\ell+1} \int_{\Omega} \phi\left(x, r_{n}^{i-1}\right) S\left(p_{n}^{i-1}\right)\left[c_{n}^{i}\right]^{\ell+1} \mathrm{~d} x \\
& -\frac{1}{h} \int_{\Omega} \phi\left(x, r_{n}^{i-1}\right) S\left(p_{n}^{i-1}\right) c_{n}^{i-1}\left[c_{n}^{i}\right]^{\ell} \mathrm{d} x \\
& +\int_{\Omega} \ell\left[c_{n}^{i}\right]^{\ell-1} \phi\left(x, r_{n}^{i-1}\right) S\left(p_{n}^{i-1}\right) D_{w}\left(p_{n}^{i-1}\right) \nabla c_{n}^{i} \cdot \nabla c_{n}^{i} \mathrm{~d} x \\
& =0 .
\end{aligned}
$$

Applying the Young's inequality, for the term in the fifth line in 4.31) we can write

$$
\begin{aligned}
& \frac{1}{h} \int_{\Omega} \phi\left(x, r_{n}^{i-1}\right) S\left(p_{n}^{i-1}\right) c_{n}^{i-1}\left[c_{n}^{i}\right]^{\ell} \mathrm{d} x \\
\leq & \frac{1}{h} \frac{1}{\ell+1} \int_{\Omega} \phi\left(x, r_{n}^{i-1}\right) S\left(p_{n}^{i-1}\right)\left[c_{n}^{i-1}\right]^{\ell+1} \mathrm{~d} x \\
& +\frac{1}{h} \frac{\ell}{\ell+1} \int_{\Omega} \phi\left(x, r_{n}^{i-1}\right) S\left(p_{n}^{i-1}\right)\left[c_{n}^{i}\right]^{\ell+1} \mathrm{~d} x .
\end{aligned}
$$

Taking (4.31) and (4.32) together we deduce

$$
\begin{aligned}
& \frac{1}{h} \frac{1}{\ell+1} \int_{\Omega} \phi\left(x, r_{n}^{i}\right) S\left(p_{n}^{i}\right)\left[c_{n}^{i}\right]^{\ell+1} \mathrm{~d} x \\
& -\frac{1}{h} \frac{1}{\ell+1} \int_{\Omega} \phi\left(x, r_{n}^{i-1}\right) S\left(p_{n}^{i-1}\right)\left[c_{n}^{i-1}\right]^{\ell+1} \mathrm{~d} x \\
& +\int_{\Omega} \ell\left[c_{n}^{i}\right]^{\ell-1} \phi\left(x, r_{n}^{i-1}\right) S\left(p_{n}^{i-1}\right) D_{w}\left(p_{n}^{i-1}\right)\left|\nabla c_{n}^{i}\right|^{2} \mathrm{~d} x \\
& \leq 0 .
\end{aligned}
$$


Now, we sum (4.33) for $i=1, \ldots, j$ to get

$$
\begin{aligned}
& \int_{\Omega} \phi\left(x, r_{n}^{j}\right) S\left(p_{n}^{j}\right)\left[c_{n}^{j}\right]^{\ell+1} \mathrm{~d} x \\
& +h \sum_{i=1}^{j} \int_{\Omega} \ell(\ell+1)\left[c_{n}^{i}\right]^{\ell-1} \phi\left(x, r_{n}^{i-1}\right) S\left(p_{n}^{i-1}\right) D_{w}\left(p_{n}^{i-1}\right)\left|\nabla c_{n}^{i}\right|^{2} \mathrm{~d} x \\
\leq & \int_{\Omega} \phi\left(x, r_{n}^{0}\right) S\left(p_{n}^{0}\right)\left[c_{n}^{0}\right]^{\ell+1} \mathrm{~d} x .
\end{aligned}
$$

Note that the second integral in (4.34) is nonnegative ( $\ell$ is supposed to be the odd integer). Moreover, in view of (4.34), (2.12) and (4.6) we have

$$
\left\|c_{n}^{j}\right\|_{L^{\ell+1}(\Omega)} \leq C\left\|c_{0}\right\|_{L^{\ell+1}(\Omega)},
$$

where the constant $C$ is independent of $\ell$ and $n$. Now, letting $\ell \rightarrow+\infty$ in (4.35), we obtain

$$
\left\|c_{n}^{j}\right\|_{L^{\infty}(\Omega)} \leq C, \quad j=1, \ldots, n .
$$

The same $L^{\infty}$-estimate can be drawn for temperature approximations, i.e.

$$
\left\|\vartheta_{n}^{j}\right\|_{L^{\infty}(\Omega)} \leq C, \quad j=1, \ldots, n .
$$

Because many steps of the proof of (4.37) are similar to those from the preceding estimate (4.36), we shall proceed more rapidly here, without explaining particular steps once more.

At the same time, from (4.4) and using (2.13) we have

$$
\begin{aligned}
\left\|r_{n}^{i}\right\|_{L^{\infty}(\Omega)}=\| h \sum_{j=1}^{i} f\left(x, p_{n}^{j}, c_{n}^{j-1}, \vartheta_{n}^{j-1},\right. & \left.r_{n}^{j-1}\right) \|_{L^{\infty}(\Omega)} \\
& \leq i h C_{f} \leq T C_{f}, \quad i=1, \ldots, n .
\end{aligned}
$$

\subsubsection{Energy estimates for discrete approximations of primary unknowns}

We start with the uniform estimate for pressure approximations $p_{n}^{i}$. We test (4.1) with $\zeta=p_{n}^{i}$ to get

$$
\begin{aligned}
& \int_{\Omega}\left[\phi\left(x, r_{n}^{i}\right)-\phi\left(x, r_{n}^{i-1}\right)\right] S\left(p_{n}^{i}\right) p_{n}^{i} \mathrm{~d} x \\
& +\int_{\Omega} \phi\left(x, r_{n}^{i-1}\right)\left[S\left(p_{n}^{i}\right)-S\left(p_{n}^{i-1}\right)\right] p_{n}^{i} \mathrm{~d} x \\
& +h \int_{\Omega} a\left(x, p_{n}^{i}, \vartheta_{n}^{i-1}, r_{n}^{i-1}\right) \nabla p_{n}^{i} \cdot \nabla p_{n}^{i} \mathrm{~d} x \\
= & h \int_{\Omega} \alpha_{1} f\left(x, p_{n}^{i}, c_{n}^{i-1}, \vartheta_{n}^{i-1}, r_{n}^{i-1}\right) p_{n}^{i} \mathrm{~d} x .
\end{aligned}
$$


Define the function $\Theta_{S}: \mathbb{R} \rightarrow \mathbb{R}$ given by the equation

$$
\Theta_{S}(\xi)=\int_{0}^{\xi} S^{\prime}(z) z \mathrm{~d} z, \quad \xi \in \mathbb{R} .
$$

It is easy to check that

$$
\Theta_{S}\left(\xi_{1}\right)-\Theta_{S}\left(\xi_{2}\right) \leq\left[S\left(\xi_{1}\right)-S\left(\xi_{2}\right)\right] \xi_{1} \quad \forall \xi_{1}, \xi_{2} \in \mathbb{R} .
$$

Using the inequality (4.41) in the equation (4.39) we arrive at

$$
\begin{aligned}
& \int_{\Omega}\left(\phi\left(x, r_{n}^{i}\right)-\phi\left(x, r_{n}^{i-1}\right)\right)\left[S\left(p_{n}^{i}\right) p_{n}^{i}-\Theta_{S}\left(p_{n}^{i}\right)\right] \mathrm{d} x \\
& +\int_{\Omega}\left[\phi\left(x, r_{n}^{i}\right) \Theta_{S}\left(p_{n}^{i}\right)-\phi\left(x, r_{n}^{i-1}\right) \Theta_{S}\left(p_{n}^{i-1}\right)\right] \mathrm{d} x \\
& +h \int_{\Omega} a\left(x, p_{n}^{i}, \vartheta_{n}^{i-1}, r_{n}^{i-1}\right) \nabla p_{n}^{i} \cdot \nabla p_{n}^{i} \mathrm{~d} x \\
\leq & h \int_{\Omega} \alpha_{1} f\left(x, p_{n}^{i}, c_{n}^{i-1}, \vartheta_{n}^{i-1}, r_{n}^{i-1}\right) p_{n}^{i} \mathrm{~d} x .
\end{aligned}
$$

From this we have

$$
\begin{aligned}
& \int_{\Omega}\left[\phi\left(x, r_{n}^{i}\right) \Theta_{S}\left(p_{n}^{i}\right)-\phi\left(x, r_{n}^{i-1}\right) \Theta_{S}\left(p_{n}^{i-1}\right)\right] \mathrm{d} x \\
& +h \int_{\Omega} a\left(x, p_{n}^{i}, \vartheta_{n}^{i-1}, r_{n}^{i-1}\right)\left|\nabla p_{n}^{i}\right|^{2} \mathrm{~d} x \\
\leq & h \int_{\Omega} \alpha_{1} f\left(x, p_{n}^{i}, c_{n}^{i-1}, \vartheta_{n}^{i-1}, r_{n}^{i-1}\right) p_{n}^{i} \mathrm{~d} x \\
& -\int_{\Omega}\left(\phi\left(x, r_{n}^{i}\right)-\phi\left(x, r_{n}^{i-1}\right)\right)\left[S\left(p_{n}^{i}\right) p_{n}^{i}-\Theta_{S}\left(p_{n}^{i}\right)\right] \mathrm{d} x .
\end{aligned}
$$

Taking into account (2.11) and (2.13) and estimating the right-hand side in (4.42) we deduce

$$
\begin{aligned}
& h \int_{\Omega} \alpha_{1} f\left(x, p_{n}^{i}, c_{n}^{i-1}, \vartheta_{n}^{i-1}, r_{n}^{i-1}\right) p_{n}^{i} \mathrm{~d} x \\
& -\int_{\Omega}\left(\phi\left(x, r_{n}^{i}\right)-\phi\left(x, r_{n}^{i-1}\right)\right)\left[S\left(p_{n}^{i}\right) p_{n}^{i}-\Theta_{S}\left(p_{n}^{i}\right)\right] \mathrm{d} x \\
\leq & h \int_{\Omega}\left|\alpha_{1} f\left(x, p_{n}^{i}, c_{n}^{i-1}, \vartheta_{n}^{i-1}, r_{n}^{i-1}\right) p_{n}^{i}\right| \mathrm{d} x \\
& +\int_{\Omega} C_{\phi}\left|r_{n}^{i}-r_{n}^{i-1}\right|\left|S\left(p_{n}^{i}\right) p_{n}^{i}-\Theta_{S}\left(p_{n}^{i}\right)\right| \mathrm{d} x \\
\leq & \left|\alpha_{1}\right| h \int_{\Omega}\left|f\left(x, p_{n}^{i}, c_{n}^{i-1}, \vartheta_{n}^{i-1}, r_{n}^{i-1}\right)\right|\left|p_{n}^{i}\right| \mathrm{d} x \\
& +h \int_{\Omega} C_{\phi}\left|f\left(x, p_{n}^{i}, c_{n}^{i-1}, \vartheta_{n}^{i-1}, r_{n}^{i-1}\right)\right|\left|S\left(p_{n}^{i}\right) p_{n}^{i}-\Theta_{S}\left(p_{n}^{i}\right)\right| \mathrm{d} x \\
\leq & C_{1} h \int_{\Omega}\left|p_{n}^{i}\right| \mathrm{d} x+C_{2} h \int_{\Omega} \Theta_{S}\left(p_{n}^{i}\right) \mathrm{d} x .
\end{aligned}
$$


Combining (4.42) and (4.43), using (4.6) and applying the Young's inequality to the first term on the right-hand side in (4.43) we get

$$
\begin{aligned}
& \int_{\Omega}\left[\phi\left(x, r_{n}^{i}\right) \Theta_{S}\left(p_{n}^{i}\right)-\phi\left(x, r_{n}^{i-1}\right) \Theta_{S}\left(p_{n}^{i-1}\right)\right] \mathrm{d} x+C_{1} h \int_{\Omega}\left|\nabla p_{n}^{i}\right|^{2} \mathrm{~d} x \\
\leq & C_{2} h+C_{3} h \int_{\Omega} \Theta_{S}\left(p_{n}^{i}\right) \mathrm{d} x .
\end{aligned}
$$

Sum (4.44) for $i=1,2, \ldots, k$. We have

$$
\begin{aligned}
& \int_{\Omega} \phi\left(x, r_{n}^{k}\right) \Theta_{S}\left(p_{n}^{k}\right) \mathrm{d} x+C_{1} h \sum_{i=1}^{k} \int_{\Omega}\left|\nabla p_{n}^{i}\right|^{2} \mathrm{~d} x \\
\leq & \int_{\Omega} \phi\left(x, r_{n}^{0}\right) \Theta_{S}\left(p_{n}^{0}\right) \mathrm{d} x+C_{2} k h+C_{3} h \sum_{i=1}^{k} \int_{\Omega} \Theta_{S}\left(p_{n}^{i}\right) \mathrm{d} x, \quad k=1,2, \ldots, n .
\end{aligned}
$$

We now apply the discrete version of the Gronwall's inequality (see e.g. [45, Chapter 1, inequality (1.67)]) to get

$$
\int_{\Omega} \Theta_{S}\left(p_{n}^{k}\right) \mathrm{d} x+h \sum_{i=1}^{k} \int_{\Omega}\left|\nabla p_{n}^{i}\right|^{2} \mathrm{~d} x \leq C, \quad k=1,2, \ldots, n .
$$

In what follows, we proceed by proving a similar uniform estimate for approximations of $c$. Using $\eta=2 c_{n}^{i}$ as a test function in (4.2) we have

$$
\begin{aligned}
& \int_{\Omega} \phi\left(x, r_{n}^{i}\right) S\left(p_{n}^{i}\right)\left(c_{n}^{i}\right)^{2}-\phi\left(x, r_{n}^{i-1}\right) S\left(p_{n}^{i-1}\right)\left(c_{n}^{i-1}\right)^{2} \mathrm{~d} x \\
& +\int_{\Omega}\left[\phi\left(x, r_{n}^{i}\right) S\left(p_{n}^{i}\right)-\phi\left(x, r_{n}^{i-1}\right) S\left(p_{n}^{i-1}\right)\right]\left(c_{n}^{i}\right)^{2} \mathrm{~d} x \\
& +\int_{\Omega} \phi\left(x, r_{n}^{i-1}\right) S\left(p_{n}^{i-1}\right)\left(c_{n}^{i}-c_{n}^{i-1}\right)^{2} \mathrm{~d} x \\
& +2 h \int_{\Omega} \phi\left(x, r_{n}^{i}\right) S\left(p_{n}^{i}\right) D_{w}\left(x, p_{n}^{i}\right) \nabla c_{n}^{i} \cdot \nabla c_{n}^{i} \mathrm{~d} x \\
& +h \int_{\Omega} a\left(x, p_{n}^{i}, \vartheta_{n}^{i-1}, r_{n}^{i-1}\right) \nabla p_{n}^{i} \cdot 2 c_{n}^{i} \nabla c_{n}^{i} \mathrm{~d} x \\
& =0 .
\end{aligned}
$$

One is allowed to use $\zeta=\left(c_{n}^{i}\right)^{2}$ as a test function in (4.1) to obtain

$$
\begin{aligned}
& \int_{\Omega}\left[\phi\left(x, r_{n}^{i}\right) S\left(p_{n}^{i}\right)-\phi\left(x, r_{n}^{i-1}\right) S\left(p_{n}^{i-1}\right)\right]\left(c_{n}^{i}\right)^{2} \mathrm{~d} x \\
& +h \int_{\Omega} a\left(x, p_{n}^{i}, \vartheta_{n}^{i-1}, r_{n}^{i-1}\right) \nabla p_{n}^{i} \cdot \nabla\left(c_{n}^{i}\right)^{2} \mathrm{~d} x \\
= & h \int_{\Omega} \alpha_{1} f\left(x, p_{n}^{i}, c_{n}^{i-1}, \vartheta_{n}^{i-1}, r_{n}^{i-1}\right)\left(c_{n}^{i}\right)^{2} \mathrm{~d} x .
\end{aligned}
$$


Subtracting (4.48) from (4.47) gives

$$
\begin{aligned}
& \int_{\Omega} \phi\left(x, r_{n}^{i}\right) S\left(p_{n}^{i}\right)\left(c_{n}^{i}\right)^{2}-\phi\left(x, r_{n}^{i-1}\right) S\left(p_{n}^{i-1}\right)\left(c_{n}^{i-1}\right)^{2} \mathrm{~d} x \\
& +\int_{\Omega} \phi\left(x, r_{n}^{i-1}\right) S\left(p_{n}^{i-1}\right)\left(c_{n}^{i}-c_{n}^{i-1}\right)^{2} \mathrm{~d} x \\
& +2 h \int_{\Omega} \phi\left(x, r_{n}^{i}\right) S\left(p_{n}^{i}\right) D_{w}\left(x, p_{n}^{i}\right) \nabla c_{n}^{i} \cdot \nabla c_{n}^{i} \mathrm{~d} x \\
& +h \int_{\Omega} \alpha_{1} f\left(x, p_{n}^{i}, c_{n}^{i-1}, \vartheta_{n}^{i-1}, r_{n}^{i-1}\right)\left(c_{n}^{i}\right)^{2} \mathrm{~d} x \\
& =0 .
\end{aligned}
$$

Upon addition (4.49) for $i=1,2, \ldots, j$ and taking into account (2.13), we can write

$$
\begin{aligned}
& \int_{\Omega} \phi\left(x, r_{n}^{j}\right) S\left(p_{n}^{j}\right)\left(c_{n}^{j}\right)^{2} \mathrm{~d} x \\
& \quad \leq \phi\left(x, r_{n}^{0}\right) S\left(p_{n}^{0}\right)\left(c_{n}^{0}\right)^{2}+h\left|\alpha_{1}\right| C_{f} \sum_{i=1}^{j} \int_{\Omega}\left(c_{n}^{i}\right)^{2} \mathrm{~d} x, \quad j=1, \ldots, n .
\end{aligned}
$$

Noting that, in view of (4.6), (2.1), (2.9) and (2.12), there exists a positive constant $C$ (independent of $n$ ) such that

$$
\phi\left(x, r_{n}^{i}\right), S\left(p_{n}^{i}\right), D_{w}\left(x, p_{n}^{i}\right)>C \quad \text { in } \Omega, \quad i=1, \ldots, n,
$$

the inequality (4.50) can be simplified to

$$
\int_{\Omega}\left(c_{n}^{j}\right)^{2} \mathrm{~d} x \leq C_{1}+C_{2} h \sum_{i=1}^{j} \int_{\Omega}\left(c_{n}^{i}\right)^{2} \mathrm{~d} x, \quad j=1, \ldots, n .
$$

Now, similarly as in (4.45), we can use the Gronwall's inequality. By doing that, in view of (4.49), (4.51) and (4.52), we obtain the estimate

$$
\max _{i=1, \ldots, n} \int_{\Omega}\left|c_{n}^{i}\right|^{2} \mathrm{~d} x+h \sum_{i=1}^{n} \int_{\Omega}\left|\nabla c_{n}^{i}\right|^{2} \mathrm{~d} x \leq C .
$$

The same uniform estimate can be drawn for the temperature approximations $\vartheta_{n}^{i}$. We use $\psi=2 \vartheta_{n}^{i}$ as a test function in (4.3) to obtain

$$
\begin{aligned}
& \quad \int_{\Omega} \frac{\phi\left(x, r_{n}^{i}\right) S\left(p_{n}^{i}\right) \vartheta_{n}^{i}-\phi\left(x, r_{n}^{i-1}\right) S\left(p_{n}^{i-1}\right) \vartheta_{n}^{i-1}}{h} 2 \vartheta_{n}^{i} \mathrm{~d} x \\
& +\int_{\Omega} \frac{\varrho\left(x, r_{n}^{i}\right) \vartheta_{n}^{i}-\varrho\left(x, r_{n}^{i-1}\right) \vartheta_{n}^{i-1}}{h} 2 \vartheta_{n}^{i} \mathrm{~d} x \\
& +2 \int_{\Omega} \lambda\left(x, p_{n}^{i-1}, \vartheta_{n}^{i-1}, r_{n}^{i-1}\right)\left|\nabla \vartheta_{n}^{i}\right|^{2} \mathrm{~d} x \\
& +\int_{\Omega} a\left(x, p_{n}^{i}, \vartheta_{n}^{i-1}, r_{n}^{i-1}\right) \nabla p_{n}^{i} \cdot 2 \vartheta_{n}^{i} \nabla \vartheta_{n}^{i} \mathrm{~d} x \\
& =2 \int_{\Omega} \alpha_{2} f\left(x, p_{n}^{i}, c_{n}^{i-1}, \vartheta_{n}^{i-1}, r_{n}^{i-1}\right) \vartheta_{n}^{i} \mathrm{~d} x .
\end{aligned}
$$


The above equation may be written as

$$
\begin{aligned}
& \int_{\Omega} \frac{\phi\left(x, r_{n}^{i}\right) S\left(p_{n}^{i}\right)\left[\vartheta_{n}^{i}\right]^{2}-\phi\left(x, r_{n}^{i-1}\right) S\left(p_{n}^{i-1}\right)\left[\vartheta_{n}^{i-1}\right]^{2}}{h} \mathrm{~d} x \\
& +\int_{\Omega} \frac{\phi\left(x, r_{n}^{i}\right) S\left(p_{n}^{i}\right)-\phi\left(x, r_{n}^{i-1}\right) S\left(p_{n}^{i-1}\right)}{h}\left[\vartheta_{n}^{i}\right]^{2} \mathrm{~d} x \\
& +\int_{\Omega} \frac{\varrho\left(x, r_{n}^{i}\right)\left[\vartheta_{n}^{i}\right]^{2}-\varrho\left(x, r_{n}^{i-1}\right)\left[\vartheta_{n}^{i-1}\right]^{2}}{h} \mathrm{~d} x+\int_{\Omega} \frac{\varrho\left(x, r_{n}^{i}\right)-\varrho\left(x, r_{n}^{i-1}\right)}{h}\left[\vartheta_{n}^{i}\right]^{2} \mathrm{~d} x \\
& +\int_{\Omega} \frac{\phi\left(x, r_{n}^{i-1}\right) S\left(p_{n}^{i-1}\right)\left[\vartheta_{n}^{i}-\vartheta_{n}^{i-1}\right]^{2}}{h} \mathrm{~d} x+\int_{\Omega} \frac{\varrho\left(x, r_{n}^{i-1}\right)\left[\vartheta_{n}^{i}-\vartheta_{n}^{i-1}\right]^{2}}{h} \mathrm{~d} x \\
& +2 \int_{\Omega} \lambda\left(x, p_{n}^{i-1}, \vartheta_{n}^{i-1}, r_{n}^{i-1}\right)\left|\nabla \vartheta_{n}^{i}\right|^{2} \mathrm{~d} x \\
& +\int_{\Omega} a\left(x, p_{n}^{i}, \vartheta_{n}^{i-1}, r_{n}^{i-1}\right) \nabla p_{n}^{i} \cdot \nabla\left[\vartheta_{n}^{i}\right]^{2} \mathrm{~d} x \\
& =2 \int_{\Omega} \alpha_{2} f\left(x, p_{n}^{i}, c_{n}^{i-1}, \vartheta_{n}^{i-1}, r_{n}^{i-1}\right) \vartheta_{n}^{i} \mathrm{~d} x .
\end{aligned}
$$

Putting $\zeta=\left[\vartheta_{n}^{i}\right]^{2}$ into 4.1, we get

$$
\begin{aligned}
& \int_{\Omega} \frac{\phi\left(x, r_{n}^{i}\right) S\left(p_{n}^{i}\right)-\phi\left(x, r_{n}^{i-1}\right) S\left(p_{n}^{i-1}\right)}{h}\left[\vartheta_{n}^{i}\right]^{2} \mathrm{~d} x \\
& +\int_{\Omega} a\left(x, p_{n}^{i}, \vartheta_{n}^{i-1}, r_{n}^{i-1}\right) \nabla p_{n}^{i} \cdot \nabla\left[\vartheta_{n}^{i}\right]^{2} \mathrm{~d} x \\
= & \int_{\Omega} \alpha_{1} f\left(x, p_{n}^{i}, c_{n}^{i-1}, \vartheta_{n}^{i-1}, r_{n}^{i-1}\right)\left[\vartheta_{n}^{i}\right]^{2} \mathrm{~d} x .
\end{aligned}
$$

Substituting (4.55) into (4.54), multiplying by $h$ and taking into account 4.4) we deduce

$$
\begin{aligned}
& \int_{\Omega}\left(\phi\left(x, r_{n}^{i}\right) S\left(p_{n}^{i}\right)\left[\vartheta_{n}^{i}\right]^{2}-\phi\left(x, r_{n}^{i-1}\right) S\left(p_{n}^{i-1}\right)\left[\vartheta_{n}^{i-1}\right]^{2}\right) \mathrm{d} x \\
& +\int_{\Omega}\left(\varrho\left(x, r_{n}^{i}\right)\left[\vartheta_{n}^{i}\right]^{2}-\varrho\left(x, r_{n}^{i-1}\right)\left[\vartheta_{n}^{i-1}\right]^{2}\right) \mathrm{d} x \\
& +2 \int_{\Omega} \lambda\left(x, p_{n}^{i-1}, \vartheta_{n}^{i-1}, r_{n}^{i-1}\right)\left|\nabla \vartheta_{n}^{i}\right|^{2} \mathrm{~d} x \\
& \leq h \int_{\Omega}\left(2\left|\alpha_{2}\right|\left|\vartheta_{n}^{i}\right|+\left|\alpha_{1}\right|\left[\vartheta_{n}^{i}\right]^{2}+\left[\vartheta_{n}^{i}\right]^{2}\right)\left|f\left(x, p_{n}^{i}, c_{n}^{i-1}, \vartheta_{n}^{i-1}, r_{n}^{i-1}\right)\right| \mathrm{d} x .
\end{aligned}
$$

We now apply the estimate

$$
\begin{aligned}
h \int_{\Omega}\left(2\left|\alpha_{2}\right|\left|\vartheta_{n}^{i}\right|+\left|\alpha_{1}\right|\left[\vartheta_{n}^{i}\right]^{2}+\left[\vartheta_{n}^{i}\right]^{2}\right)\left|f\left(x, p_{n}^{i}, c_{n}^{i-1}, \vartheta_{n}^{i-1}, r_{n}^{i-1}\right)\right| \mathrm{d} x & \\
& \leq h \int_{\Omega}\left(C_{1}+C_{2}\left[\vartheta_{n}^{i}\right]^{2}\right) C_{f} \mathrm{~d} x
\end{aligned}
$$


which holds for "sufficiently large" $C_{1}$, and sum (4.56) for $i=1, \ldots, j$ to obtain

$$
\begin{aligned}
& \int_{\Omega}\left(\phi\left(x, r_{n}^{j}\right) S\left(p_{n}^{j}\right)+\varrho\left(x, r_{n}^{j}\right)\right)\left[\vartheta_{n}^{j}\right]^{2} \mathrm{~d} x \\
& +2 h \sum_{i=1}^{j} \int_{\Omega} \lambda\left(x, p_{n}^{i-1}, \vartheta_{n}^{i-1}, r_{n}^{i-1}\right)\left|\nabla \vartheta_{n}^{i}\right|^{2} \mathrm{~d} x \\
& \leq \int_{\Omega}\left(\phi\left(x, r_{n}^{0}\right) S\left(p_{n}^{0}\right)+\varrho\left(x, r_{n}^{0}\right)\right)\left[\vartheta_{n}^{0}\right]^{2} \mathrm{~d} x \\
& +h \sum_{i=1}^{j} \int_{\Omega}\left(C_{1}+C_{2}\left[\vartheta_{n}^{i}\right]^{2}\right) C_{f} \mathrm{~d} x .
\end{aligned}
$$

By (2.7), (2.10), (2.12) and (4.6), the above estimate may be simplified as

$$
\int_{\Omega}\left|\vartheta_{n}^{j}\right|^{2} \mathrm{~d} x+h \sum_{i=1}^{j} \int_{\Omega}\left|\nabla \vartheta_{n}^{i}\right|^{2} \mathrm{~d} x \leq C_{1}+C_{2} T+C_{3} h \sum_{i=1}^{j} \int_{\Omega}\left|\vartheta_{n}^{i}\right|^{2} \mathrm{~d} x .
$$

As before, we can now use the Gronwall's inequality. As a consequence, we obtain

$$
\int_{\Omega}\left|\vartheta_{n}^{j}\right|^{2} \mathrm{~d} x+h \sum_{i=1}^{j} \int_{\Omega}\left|\nabla \vartheta_{n}^{i}\right|^{2} \mathrm{~d} x \leq C, \quad j=1,2, \ldots, n .
$$

Finally, using Assumptions (iv) and (vi), (4.46), (4.53) and (4.57) and applying [45, Proposition 1.28], the uniform estimate

$$
h \sum_{i=1}^{j} \int_{\Omega}\left|\nabla r_{n}^{i}\right|^{2} \mathrm{~d} x \leq C, \quad j=1,2, \ldots, n,
$$

can be obtained directly from (4.4). Note that (4.58) together with (4.38) yields

$$
h \sum_{i=1}^{j} \int_{\Omega}\left\|r_{n}^{i}\right\|_{W^{1,2}(\Omega)}^{2} \mathrm{~d} x \leq C, \quad j=1,2, \ldots, n .
$$

Moreover, from (4.4) (see also 4.29) one observes immediately that

$$
\frac{r_{n}^{i}-r_{n}^{i-1}}{h}=f\left(x, p_{n}^{i}, c_{n}^{i-1}, \vartheta_{n}^{i-1}, r_{n}^{i-1}\right)
$$

and therefore, in view of 2.13, we have

$$
\left\|\frac{r_{n}^{i}-r_{n}^{i-1}}{h}\right\|_{L^{\infty}(\Omega)} \leq C_{f}, \quad i=1,2, \ldots, n
$$

\subsubsection{Further estimates}

We now derive additional auxiliary estimates which will be used in the following section. Such estimates play the crucial role in compactness arguments (see [2, Lemma 1.9]) and taking the limit $n \rightarrow+\infty$. 
Let us sum up (4.1) for $i=j+1, \ldots, j+k$ and then put $\zeta=p_{n}^{j+k}-p_{n}^{j}$. This leads to

$$
\begin{aligned}
& \int_{\Omega}\left[\phi\left(x, r_{n}^{j+k}\right) S\left(p_{n}^{j+k}\right)-\phi\left(x, r_{n}^{j}\right) S\left(p_{n}^{j}\right)\right]\left(p_{n}^{j+k}-p_{n}^{j}\right) \mathrm{d} x \\
+ & h \sum_{i=j+1}^{j+k} \int_{\Omega} a\left(x, p_{n}^{i}, \vartheta_{n}^{i-1}, r_{n}^{i-1}\right) \nabla p_{n}^{i} \cdot \nabla\left(p_{n}^{j+k}-p_{n}^{j}\right) \mathrm{d} x \\
= & h \sum_{i=j+1}^{j+k} \int_{\Omega} \alpha_{1} f\left(x, p_{n}^{i}, c_{n}^{i-1}, \vartheta_{n}^{i-1}, r_{n}^{i-1}\right)\left(p_{n}^{j+k}-p_{n}^{j}\right) \mathrm{d} x .
\end{aligned}
$$

From this and in view of (2.8) and (2.13) we have

$$
\begin{aligned}
& \int_{\Omega}\left[\phi\left(x, r_{n}^{j+k}\right) S\left(p_{n}^{j+k}\right)-\phi\left(x, r_{n}^{j}\right) S\left(p_{n}^{j}\right)\right]\left(p_{n}^{j+k}-p_{n}^{j}\right) \mathrm{d} x \\
\leq & C_{1} h \sum_{i=j+1}^{j+k} \int_{\Omega}\left|\nabla p_{n}^{i}\right|\left|\nabla\left(p_{n}^{j+k}-p_{n}^{j}\right)\right| \mathrm{d} x \\
& +k h\left|\alpha_{1}\right| C_{f} \int_{\Omega}\left|p_{n}^{j+k}-p_{n}^{j}\right| \mathrm{d} x .
\end{aligned}
$$

Using (2.11) and (4.4), the above estimate can be further rewritten as

$$
\begin{aligned}
& \int_{\Omega} \phi\left(x, r_{n}^{j+k}\right)\left[S\left(p_{n}^{j+k}\right)-S\left(p_{n}^{j}\right)\right]\left(p_{n}^{j+k}-p_{n}^{j}\right) \mathrm{d} x \\
\leq & C_{1} h \sum_{i=j+1}^{j+k} \int_{\Omega}\left|\nabla p_{n}^{i} \| \nabla\left(p_{n}^{j+k}-p_{n}^{j}\right)\right| \mathrm{d} x \\
& +\left(k h\left|\alpha_{1}\right| C_{f}+k h S_{s} C_{\phi} C_{f}\right) \int_{\Omega}\left|p_{n}^{j+k}-p_{n}^{j}\right| \mathrm{d} x .
\end{aligned}
$$

Again, we sum (4.63) for $j=1, \ldots, n-k$, multiply it by $h$ and use (2.12) and (4.46) to arrive at

$$
h \sum_{j=1}^{n-k} \int_{\Omega}\left[S\left(p_{n}^{j+k}\right)-S\left(p_{n}^{j}\right)\right]\left(p_{n}^{j+k}-p_{n}^{j}\right) \mathrm{d} x \leq C k h, \quad 0 \leq k<n .
$$

Further, using the same arguments as in (4.61)-(4.64), we arrive at

$$
h \sum_{j=1}^{n-k} \int_{\Omega}\left|c_{n}^{j+k}-c_{n}^{j}\right|^{2} \mathrm{~d} x \leq C k h .
$$

Finally, derivation similar to that presented above leads to

$$
h \sum_{j=1}^{n-k} \int_{\Omega}\left|\vartheta_{n}^{j+k}-\vartheta_{n}^{j}\right|^{2} \mathrm{~d} x \leq C k h
$$


At the same time, it is easily deduced from (4.29) that

$$
h \sum_{j=1}^{n-k} \int_{\Omega}\left|r_{n}^{j+k}-r_{n}^{j}\right|^{2} \mathrm{~d} x \leq C k h .
$$

\subsection{Temporal interpolants and uniform estimates}

By means of the sequences $p_{n}^{i}, c_{n}^{i}, \vartheta_{n}^{i}, r_{n}^{i}$ constructed in Section 4.1, we define the piecewise constant interpolants $\bar{\varphi}_{n}(t)=\varphi_{n}^{i}$ for $t \in((i-1) h, i h]$ and, in addition, we extend $\bar{\varphi}_{n}$ for $t \leq 0$ by $\bar{\varphi}_{n}(t)=\varphi_{0}$ for $t \in(-h, 0]$. Here, $\varphi_{n}^{i}$ stands for $p_{n}^{i}, c_{n}^{i}, \vartheta_{n}^{i}$ or $r_{n}^{i}$.

For a function $\varphi$ we often use the simplified notation $\varphi:=\varphi(t), \varphi_{h}(t):=\varphi(t-h)$, $\partial_{t}^{-h} \varphi(t):=\frac{\varphi(t)-\varphi(t-h)}{h}, \partial_{t}^{h} \varphi(t):=\frac{\varphi(t+h)-\varphi(t)}{h}$. Then, following (4.1)-4.3), the piecewise constant time interpolants $\bar{p}_{n} \in L^{\infty}\left(I ; W_{\Gamma_{D}}^{1, s}(\Omega)\right), \bar{c}_{n} \in L^{\infty}\left(I ; W_{\Gamma_{D}}^{1, s}(\Omega)\right)$ and $\bar{\vartheta}_{n} \in L^{\infty}\left(I ; W_{\Gamma_{D}}^{1, s}(\Omega)\right)$ (with some $s>2$ ) satisfy the equations

$$
\begin{aligned}
& \int_{\Omega} \partial_{t}^{-h}\left[\phi\left(x, \bar{r}_{n}(t)\right) S\left(\bar{p}_{n}(t)\right)\right] \zeta \mathrm{d} x \\
& +\int_{\Omega} a\left(x, \bar{p}_{n}(t), \bar{\vartheta}_{n}(t-h), \bar{r}_{n}(t-h)\right) \nabla \bar{p}_{n}(t) \cdot \nabla \zeta \mathrm{d} x \\
= & \left.\left.\int_{\Omega} \alpha_{1} f\left(x, \bar{p}_{n}(t), \bar{c}_{n}(t-h), \bar{\vartheta}_{n}(t-h)\right), \bar{r}_{n}(t-h)\right)\right) \zeta \mathrm{d} x
\end{aligned}
$$

for any $\zeta \in W_{\Gamma_{D}}^{1,2}(\Omega)$,

$$
\begin{aligned}
& \int_{\Omega} \partial_{t}^{-h}\left[\phi\left(x, \bar{r}_{n}(t)\right) S\left(\bar{p}_{n}(t)\right) \bar{c}_{n}(t)\right] \eta \mathrm{d} x \\
& +\int_{\Omega} \phi\left(x, \bar{r}_{n}(t)\right) S\left(\bar{p}_{n}(t)\right) D_{w}\left(\bar{p}_{n}(t-h)\right) \nabla \bar{c}_{n}(t) \cdot \nabla \eta \mathrm{d} x \\
& +\int_{\Omega} \bar{c}_{n}(t) a\left(x, \bar{p}_{n}(t), \bar{\vartheta}_{n}(t-h), \bar{r}_{n}(t-h)\right) \nabla \bar{p}_{n}(t) \cdot \nabla \eta \mathrm{d} x \\
& =0
\end{aligned}
$$

for any $\eta \in W_{\Gamma_{D}}^{1,2}(\Omega)$ and

$$
\begin{aligned}
& \int_{\Omega} \partial_{t}^{-h}\left[\phi\left(x, \bar{r}_{n}(t)\right) S\left(\bar{p}_{n}(t)\right) \bar{\vartheta}_{n}(t)+\varrho\left(x, \bar{r}_{n}(t)\right) \bar{\vartheta}_{n}(t)\right] \psi \mathrm{d} x \\
& +\int_{\Omega} \lambda\left(x, \bar{p}_{n}(t-h), \bar{\vartheta}_{n}(t-h), \bar{r}_{n}(t-h)\right) \nabla \bar{\vartheta}_{n}(t) \cdot \nabla \psi \mathrm{d} x \\
& +\int_{\Omega} \bar{\vartheta}_{n}(t) a\left(x, \bar{p}_{n}(t), \bar{\vartheta}_{n}(t-h), \bar{r}_{n}(t-h)\right) \nabla \bar{p}_{n}(t) \cdot \nabla \psi \mathrm{d} x \\
= & \left.\left.\int_{\Omega} \alpha_{2} f\left(x, \bar{p}_{n}(t), \bar{c}_{n}(t-h), \bar{\vartheta}_{n}(t-h)\right), \bar{r}_{n}(t-h)\right)\right) \zeta \mathrm{d} x
\end{aligned}
$$


for any $\psi \in W_{\Gamma_{D}}^{1,2}(\Omega)$. Finally, from (4.4) and (4.5) we have

$$
\left.\left.\bar{R}_{n}(t)=f\left(x, \bar{p}_{n}(t), \bar{c}_{n}(t-h), \bar{\vartheta}_{n}(t-h)\right), \bar{r}_{n}(t-h)\right)\right)
$$

for all $t \in[0, T]$, where

$$
\bar{R}_{n}(t)=\frac{r_{n}^{i}-r_{n}^{i-1}}{h} \quad \text { for } t \in((i-1) h, i h], \quad i=1,2, \ldots, n
$$

and $\bar{R}_{n}(0)=r_{n}^{1} / h, r_{n}^{0}=0$. To be able to say something about the behaviour of the sequences $\left\{\bar{p}_{n}\right\},\left\{\bar{c}_{n}\right\},\left\{\bar{\vartheta}_{n}\right\},\left\{\bar{R}_{n}\right\}$, and $\left\{\bar{r}_{n}\right\}$, we now present some apriori estimates for solutions of the problem (4.68)-(4.71).

To this aim, from (4.36), (4.37), 4.38), 4.46, 4.53), (4.57) and (4.58) we see immediately that

$$
\begin{array}{r}
\sup _{0 \leq t \leq T} \int_{\Omega} \Theta_{S}\left(\bar{p}_{n}(t)\right) \mathrm{d} x+\int_{0}^{T}\left\|\bar{p}_{n}(t)\right\|_{W_{\Gamma_{D}}^{1,2}(\Omega)}^{2} \mathrm{~d} t \leq C, \\
\int_{0}^{T}\left\|\bar{c}_{n}(t)\right\|_{W_{\Gamma_{D}}^{1,2}(\Omega)}^{2} \mathrm{~d} t \leq C, \\
\int_{0}^{T}\left\|\bar{\vartheta}_{n}(t)\right\|_{W_{\Gamma_{D}}^{1,2}(\Omega)}^{2} \mathrm{~d} t \leq C, \\
\int_{0}^{T}\left\|\bar{r}_{n}(t)\right\|_{W_{\Gamma_{D}}^{1,2}(\Omega)}^{2} \mathrm{~d} t \leq C, \\
\left\|\bar{c}_{n}\right\|_{L^{\infty}\left(Q_{T}\right)} \leq C, \\
\left\|\bar{\vartheta}_{n}\right\|_{L^{\infty}\left(Q_{T}\right)} \leq C, \\
\left\|\bar{r}_{n}\right\|_{L^{\infty}\left(Q_{T}\right)} \leq C .
\end{array}
$$

Moreover, the estimates (4.64)-4.67) can be rewritten in the form

$$
\begin{aligned}
& \int_{0}^{T-k h}\left[S\left(\bar{p}_{n}(t+k h)\right)-S\left(\bar{p}_{n}(t)\right)\right]\left(\bar{p}_{n}(t+k h)-\bar{p}_{n}(t)\right) \mathrm{d} t \leq C k h, \\
& \int_{0}^{T-k h}\left|\bar{c}_{n}(t+k h)-\bar{c}_{n}(t)\right|^{2} \mathrm{~d} t \leq C k h, \\
& \int_{0}^{T-k h}\left|\bar{\vartheta}_{n}(t+k h)-\bar{\vartheta}_{n}(t)\right|^{2} \mathrm{~d} t \leq C k h, \\
& \int_{0}^{T-k h}\left|\bar{r}_{n}(t+k h)-\bar{r}_{n}(t)\right|^{2} \mathrm{~d} t \leq C k h .
\end{aligned}
$$

Now we are ready to complete the proof of the main result of this paper which is the conclusion of the following section.

\subsection{Passage to the limit}

The a-priori estimates (4.72)-(4.78) allow us to conclude that there exist $p \in L^{2}\left(I ; W_{\Gamma_{D}}^{1,2}(\Omega)\right)$, $c \in L^{2}\left(I ; W_{\Gamma_{D}}^{1,2}(\Omega)\right) \cap L^{\infty}\left(Q_{T}\right), \vartheta \in L^{2}\left(I ; W_{\Gamma_{D}}^{1,2}(\Omega)\right) \cap L^{\infty}\left(Q_{T}\right)$ and $r \in L^{\infty}\left(Q_{T}\right)$, 
such that, letting $n \rightarrow+\infty$ (along a selected subsequence),

$$
\begin{array}{ll}
\bar{p}_{n} \rightarrow p & \text { weakly in } L^{2}\left(I ; W_{\Gamma_{D}}^{1,2}(\Omega)\right), \\
\bar{c}_{n} \rightarrow c & \text { weakly in } L^{2}\left(I ; W_{\Gamma_{D}}^{1,2}(\Omega)\right), \\
\bar{c}_{n} \rightarrow c & \text { weakly star in } L^{\infty}\left(Q_{T}\right), \\
\bar{\vartheta}_{n} \rightarrow \vartheta & \text { weakly in } L^{2}\left(I ; W_{\Gamma_{D}}^{1,2}(\Omega)\right), \\
\bar{\vartheta}_{n} \rightarrow \vartheta & \text { weakly star in } L^{\infty}\left(Q_{T}\right), \\
\bar{r}_{n} \rightarrow r & \text { weakly in } L^{2}\left(I ; W^{1,2}(\Omega)\right), \\
\bar{r}_{n} \rightarrow r & \text { weakly star in } L^{\infty}\left(Q_{T}\right) .
\end{array}
$$

Thus, we derived fundamental properties of the functions $p, c, \vartheta$ and $r$. The crucial step to ensure that $p, c, \vartheta$ and $r$ solve the problem (3.1)-(3.4) consists in showing that the sequences $\left\{\bar{p}_{n}\right\},\left\{\bar{c}_{n}\right\},\left\{\bar{\vartheta}_{n}\right\}$ and $\left\{\bar{r}_{n}\right\}$, converge not only weakly in appropriate Bochner spaces, but even almost everywhere on $Q_{T}$.

To this aim, in view of (4.72) and (4.79), using the compactness argument one can show in the same way as in [2, Lemma 1.9] and [10, Eqs. (2.10)-(2.12)] that

$$
S\left(\bar{p}_{n}\right) \rightarrow S(p) \quad \text { in } L^{1}\left(Q_{T}\right)
$$

and almost everywhere on $Q_{T}$. Since $S$ is strictly monotone, it follows from (4.83) that [23, Proposition 3.35]

$$
\bar{p}_{n} \rightarrow p \quad \text { almost everywhere on } Q_{T} \text {. }
$$

By similar arguments, using estimates (4.73)-4.78) and (4.80)-4.82), we have

$$
\begin{array}{ll}
\bar{c}_{n} \rightarrow c & \text { almost everywhere on } Q_{T}, \\
\bar{\vartheta}_{n} \rightarrow \vartheta & \text { almost everywhere on } Q_{T}, \\
\bar{r}_{n} \rightarrow r & \text { almost everywhere on } Q_{T} .
\end{array}
$$

Finally, in consequence of (4.60), the norms $\left\|\bar{R}_{n}(t)\right\|_{L^{\infty}(\Omega)}$ are uniformly bounded with respect to $n$ and $t$. Hence, there exists $R \in L^{2}\left(I, L^{\infty}(\Omega)\right)$, such that

$$
\bar{R}_{n} \rightarrow R \quad \text { weakly star in } L^{2}\left(I ; L^{\infty}(\Omega)\right)
$$

and $R$ can be shown to satisfy (see [44, Chapter 11])

$$
\int_{0}^{t} R(s) \mathrm{d} s=r(t)
$$

and

$$
R(t)=r^{\prime}(t) \text { in } L^{2}\left(I ; L^{\infty}(\Omega)\right) .
$$

It follows that

$$
r \in C\left([0, T] ; L^{\infty}(\Omega)\right) \quad\left(\text { and even } A C\left([0, T] ; L^{\infty}(\Omega)\right)\right)
$$


and $r(0)=0$. In view of 4.84-4.87 and the assumption (iv) we have

$$
\left.\left.\left.\left.f\left(x, \bar{p}_{n}(t), \bar{c}_{n}(t-h), \bar{\vartheta}_{n}(t-h)\right), \bar{r}_{n}(t-h)\right)\right) \rightarrow f(x, p(t), c(t), \vartheta(t)), r(t)\right)\right)
$$

almost everywhere on $Q_{T}$ and on account of (4.71) and 4.88) we can write

$$
R=f \text { in } L^{2}\left(I ; L^{\infty}(\Omega)\right) .
$$

This leads to (3.4). Moreover, the above established convergences are sufficient for taking the limit $n \rightarrow \infty$ in (4.68)-4.70) (along a selected subsequence) to get the weak solution of the system (1.1)-1.8) in the sense of Definition 3.1 This completes the proof of the main result stated by Theorem 3.2 .

\section{Acknowledgment}

The first author of this work has been supported by the project GAČR 16-20008S. The second author of this work has been supported by the Croatian Science Foundation (scientific project 3955: Mathematical modeling and numerical simulations of processes in thin or porous domains).

\section{References}

[1] A. Adams, J.F. Fournier, Sobolev spaces, Pure and Applied Mathematics 140, Academic Press, 2003.

[2] H.W. Alt, S. Luckhaus, Quasilinear elliptic-parabolic differential equations, Mathematische Zeitschrift, 183 (1983) 311-341.

[3] J. Bear, Dynamics of Fluids in Porous Media, Courier Corporation, 1972.

[4] M. Beněs, L. Krupička, Weak solutions of coupled dual porosity flows in fractured rock mass and structured porous media, Journal of Mathematical Analysis and Applications, 433 (2016) 543-565.

[5] M. Beneš, I. Pažanin, On existence, regularity and uniqueness of thermally coupled incompressible flows in a system of three dimensional pipes, Nonlinear Analysis, 149 (2017) 56-80.

[6] M. Beneš, I. Pažanin, Homogenization of degenerate coupled fluid flows and heat transport through porous media, Journal of Mathematical Analysis and Applications, 446 (2017) 165-192.

[7] M. Beneš, J. Zeman, Some properties of strong solutions to nonlinear heat and moisture transport in multi-layer porous structures, Nonlinear Analysis: Real World Applications, 13 (2012) 1562-1580.

[8] P. Degond, S. Génieys, A. Jüngel, A system of parabolic equations in nonequilibrium thermodynamics including thermal and electrical effects, Journal de Mathématiques Pures et Appliquées, 76 (1997) 991-1015. 
[9] A. Doktor, On the solution of the heat equation with nonlinear unbounded memory, Applications of Mathematics, 30 (1985) 461-474.

[10] J. Filo, J. Kačur, Local existence of general nonlinear parabolic systems, Nonlinear Analysis, 24 (1995) 1597-1618.

[11] J. Filo, On solutions of a perturbed fast diffusion equation, Aplikace matematiky, 32 (1987) 364-380.

[12] T. Gallouët, A. Monier, On the regularity of solutions to elliptic equations, Rendiconti di Matematica, 19 (1999) 471-488.

[13] D. Gawin, F. Pesavento and B.A. Schrefler, Hygro-thermo-chemo-mechanical modelling of concrete at early ages and beyond. Part I: Hydration and hygrothermal phenomena, International Journal For Numerical Methods In Engineering, 67 (2006) 299-331.

[14] D. Gawin, F. Pesavento, B. Schrefler, What physical phenomena can be neglected when modelling concrete at high temperature? A comparative study. Part 1: Physical phenomena and mathematical model, International Journal of Solids and Structures, 48 (13) 1927-1944.

[15] D. Gawin, F. Pesavento, B. Schrefler, What physical phenomena can be neglected when modelling concrete at high temperature? A comparative study. Part 2: Comparison between models. International Journal of Solids and Structures, 48 (13) 1945-1961.

[16] M.T. van Genuchten, A closed form equation for predicting the hydraulic conductivity of unsaturated soil, Soil Science Society of America Journal, 44 (1980) 892-898.

[17] H. Gerke, M. Van Genuchten, A dual-porosity model for simulating the preferential movement of water and solutes in structured porous media, Water Resources Research, 29 (1993) 305-319.

[18] H. Gerke, M. Van Genuchten, Evaluation of the first order transfer term for variably saturated dual porosity flow models, Water Resources Research, 29 (1993) 1225-1238.

[19] M. Giaquinta, G. Modica;, Local existence for quasilinear parabolic systems under nonlinear boundary conditions, Annali di Matematica Pura ed Applicata, 149 (1987) 41-59.

[20] K. Gröger, A $W^{1, p}$-estimate for solutions to mixed boundary value problems for second order elliptic differential equations, Mathematische Annalen, 283 (1989) 679-687.

[21] P.A. Harris, E.N.M. Cirillo, A. Muntean, Weak solutions to Allen-Cahn-like equations modelling consolidation of porous media, IMA Journal of Applied Mathematics 82 (2017) 224-250. 
[22] A. Jüngel, Regularity and uniqueness of solutions to a parabolic system in nonequilibrium thermodynamics, Nonlinear Analysis, 41 (2000) 669-688.

[23] J. Kačur, On a solution of degenerate elliptic-parabolic systems in Orlicz-Sobolev spaces. I, Mathematische Zeitschrift, 203 (1990) 153-171.

[24] J. Kačur, Solution to strongly nonlinear parabolic problems by a linear approximation scheme, IMA Journal of Numerical Analysis, 19 (1999) 119-145.

[25] J. Kačur, Solution of Degenerate Convection-Diffusion Problems by the Method of Characteristics, SIAM Journal on Numerical Analysis, 39 (2001) 858-879.

[26] A. Kufner, O. John, S. Fučík, Function Spaces, Academia, 1977.

[27] O. A. Ladyzhenskaya, N.N. Ural'tseva, Linear and Quasilinelr Equations of Elliptic Type, Academic Press, New York, 1968.

[28] B. Li, W. Sun, Global existence of weak solution for nonisothermal multicomponent flow in porous textile media, SIAM Journal on Mathematical Analysis, 42 (2010) 3076-3102.

[29] B. Li, W. Sun, Y. Wang, Global existence of weak solution to the heat and moisture transport system in fibrous porous media, Journal of Differential Equations, 249 (2010) 2618-2642.

[30] B. Li, W. Sun, Global weak solution for a heat and sweat transport system in three-dimensional fibrous porous media with condensation/evaporation and absorption, SIAM Journal on Mathematical Analysis, 44 (2012) 1448-1473.

[31] T. Ishida, K. Maekawa, T. Kishi, Enhanced modeling of moisture equilibrium and transport in cementitious materials under arbitrary temperature and relative humidity history, Cement and Concrete Research, 37 (2007) 565-578.

[32] K. Maekawa, T. Ishida, T. Kishi, Multi-scale modeling of concrete performance, Journal of Advanced Concrete Technology, 1 (2003) 91-126.

[33] K. Maekawa, R. Chaube, T. Kishi, Modelling of concrete performance : hydration, microstructure formation, and mass transport. London ; New York : E \& FN Spon, 1999.

[34] J. Nečas, Les methodes directes en theorie des equations elliptiques, Academia, Prague 1967.

[35] S. Ning, Mathematical problems on the fluid-solute-heat flow through porous media, I. Unsaturated case, Acta Mathematicae Applicatae Sinica, 6 (1990) 135144.

[36] S. Ning, Mathematical problems on the fluid-solute-heat flow through porous media, II. Partially saturated case, Acta Mathematicae Applicatae Sinica, 6 (1990) 145-157. 
[37] S. Ning, An elliptic-parabolic system arising from the fluid-solute-heat flow through saturated porous media, Acta Mathematicae Applicatae Sinica, 6 (1990) 224-237.

[38] S. Ning, Multidimensional degenerate diffusion problem with evolutionary boundary condition: existence, uniqueness, and approximation, In: Flow in Porous Media, Volume 114 of the series ISNM International Series of Numerical Mathematics, (1993) 165-178.

[39] J. Ožbolt, G. Balabanić, G. Periškić, M. Kušter, Modelling the effect of damage on transport processes in concrete, Construction and Building Materials, 24 (2010) 1638-48.

[40] G.F. Pinder, W.G. Gray, Essentials of Multiphase Flow in Porous Media, WileyInterscience, New Jersey, 2008.

[41] V. Pluschke; Local solution of parabolic equations with strongly increasing nonlinearity by the Rothe method, Czechoslovak Mathematical Journal, 38 (1988) 642-654.

[42] V. Pluschke, Rothe's Method for Semilinear Parabolic Problems with Degeneration, Mathematische Nachrichten, 156 (1992) 283-295.

[43] V. Pluschke, Rothe's Method for Degenerate Quasilinear Parabolic Equations, In: Zuzana Dosla and J. Kuben and Jaromir Vosmansky (eds.): Proceedings of Equadiff 9, Conference on Differential Equations and Their Applications, Brno, August 25-29, 1997, [Part 3] Papers. Masaryk University, Brno, 1998. CD-ROM, pp. 247-254.

[44] K. Rektorys, The method of discretization in time and partial differential equations, Reidel Co, Dodrecht, Holland 1982.

[45] T. Roubíček, Nonlinear Partial Differential Equations With Applications. Birkhäuser, 2005.

[46] H.-W. Song, H.-J. Cho, S.-S. Park, K.-J. Byun, K. Maekawa, Early-age cracking resistance evaluation of concrete structures, Concrete Science and Engineering, 3 (2001) 63-72.

[47] J. Vala, On a system of equations of evolution with a non-symmetrical parabolic part occuring in the analysis of moisture and heat transfer in porous media, Applications of Mathematics, 47 (2002) 187-214.

[48] P. Weidemaier, Local existence for parabolic problems with fully nonlinear boundary condition; An $L_{p}$-approach, Annali di Matematica Pura ed Applicata, 160 (1991) 207-222.

[49] Z. Wu, J. Yin, Ch. Wang, Elliptic and Parabolic Equations, World Scientific, 2006. 